\title{
A new extension of the normal distribution
}

\author{
Maria do Carmo S. Lima* Gauss M. Cordeiro*
}

Edwin M. M. Ortega $\uparrow^{\dagger}$

\begin{abstract}
Providing a new distribution is always precious for statisticians. A new threeparameter distribution called the gamma normal distribution is defined and studied. Various structural properties of the new distribution are derived, including some explicit expressions for the moments, quantile and generating functions, mean deviations, probability weighted moments and two types of entropy. We also investigate the order statistics and their moments. Maximum likelihood techniques are used to fit the new model and to show its potentiality by means of two examples of real data. Based on three criteria, the proposed distribution provides a better fit then the skew-normal distribution.
\end{abstract}

Keywords: Gamma distribution; Maximum likelihood estimation; Mean deviation; Normal distribution; Quantile.

\section{Introduction}

In statistics, the normal distribution is the most popular model in applications to real data. When the number of observations is large, it can serve as an approximate distribution for other models. The probability density function (pdf) (for $\mathrm{x} \in \mathrm{R}$ ) of the normal $\mathrm{N}(\mu, \sigma)$ distribution becomes

$$
g(x ; \mu, \sigma)=\frac{1}{\sqrt{2 \pi} \sigma} \exp \left\{-\frac{(x-\mu)^{2}}{2 \sigma^{2}}\right\}=\frac{1}{\sigma} \phi\left(\frac{x-\mu}{\sigma}\right)
$$

where $-\infty<\mu<\infty$ is a location parameter and $\sigma>0$ is a scale parameter. Its cumulative distribution function (cdf) is given by $x-\mu$ )

$$
G(x ; \mu, \sigma)=\Phi\left(\frac{x-\mu}{\sigma}\right)
$$

A family of univariate distributions generated by gamma random variables was proposed by Zografos and Balakrishnan (2009) and Ristic and Balakrishnan (2011). They defined the gamma-G ("GG" for short) distribution from any baseline cdf $G(x), x \in R$, using an additional shape parameter a > 0 , by the pdf and cdf

$$
f(x)=\frac{g(x)}{\Gamma(a)}\{-\log [1-G(x)]\}^{a-1}
$$

and

$$
F(x)=\frac{1}{\Gamma(a)} \int_{0}^{-\log [1-G(x)]} t^{a-1} \mathrm{e}^{-\mathrm{t}} \mathrm{dt}=\gamma_{1}(\mathrm{a},-\log [1-\mathrm{G}(\mathrm{x})])
$$

\footnotetext{
* Address:Departamento de Estatística, Universidade Federal de Pernambuco - UFPE, Recife,Brazil. Email:gausscordeiro@uol.com.br

${ }^{\dagger}$ Address: Departamento de Ciências Exatas, Universidade de São Paulo - USP, Piracicaba, Brazil. E-mail: edwin@usp.br
} 
respectively, where $\mathrm{g}(\mathrm{x})=\mathrm{dG}(\mathrm{x}) / \mathrm{dx}, \Gamma(a)=\int_{0}^{\infty} t^{a-1} \mathrm{e}^{-\mathrm{t}} \mathrm{dt}$ is the gamma function, $\gamma(a, z)=\int_{0}^{z} t^{a-1} \mathrm{e}^{-\mathrm{t}} \mathrm{dt}$ and $\gamma_{1}(a, z)=\gamma(a, z) / \Gamma(a)$ are the incomplete gamma function and the incomplete gamma function ratio, respectively.

Each new GG distribution can be obtained from a specified $G$ distribution. For a $=1$, the $G$ distribution is a basic exemplar with a continuous crossover towards cases with different shapes ( for example, a particular combination of skewness and kurtosis). Zografos and Balakrishnan (2009) motivated the GG distribution as follows. Let $\mathrm{X}(1), \ldots, \mathrm{X}(\mathrm{n})$ be lower record values from a sequence of i.i.d. random variables from a population with pdf $g(x)$. Then, the pdf of the nth lower record value is given by (3) with $\mathrm{a}=\mathrm{n}$. A logarithmic transformation of the baseline distribution $\mathrm{G}$ transforms the random variable $X$ with density function (3) to a gamma distribution. In other words, if $X$ has the density (3), then the random variable $Z=-\log [1-G(X)]$ has a gamma density $\pi(z ; a)=\Gamma(a)-1 z a-1 e-z, z>0$, say $Z \sim G(a, 1)$. The opposite is also true, if $Z \sim G(a, 1)$, then the random variable $X=G-1(1-\mathrm{e}-Z)$ has the GG density function (3). Nadarajah et al. (2013) derived some mathematical properties of (3) in the most simple, explicit and general forms for any $\mathrm{G}$ distribution.

In this paper, we study some structural properties of the gamma normal (GN) distribution, which generalizes the normal disribution. In Section 2, we introduce the GN distribution and provide plots of its density function. We derive expansions for the pdf and cdf (Section 3) and explicit expressions for the quantile function (Section 4), ordinary and incomplete moments and Bonferroni and Lorenz curves (Section 5), generating function (Section 6) and entropies (Section 7). In Section 8, we investigate the order statistics and their moments. The estimation of the model parameters is performed by maximum likelihood in Section 9 and two applications are provided in Section 10. Concluding remarks are addressed in Section 11.

\section{The GN distribution}

By taking the pdf (1) and cdf (2) of the normal distribution with location parameter $\mu \in \mathrm{R}$ and dispersion parameter $\sigma>0$, the pdf and cdf of the GN distribution are obtained from equations (3) and (4) ( for $\mathrm{x}$ $\in \mathrm{R})$ as

$$
f(x)=\frac{1}{\sigma \Gamma(a)} \phi\left(\frac{x-\mu}{\sigma}\right)\left\{-\log \left[1-\Phi\left(\frac{x-\mu}{\sigma}\right)\right]\right\}^{a-1}
$$

and

$$
F(x)=\frac{1}{\Gamma(a)} \int_{0}^{-\log \left[1-\Phi\left(\frac{x-\mu}{\sigma}\right)\right]} t^{a-1} \mathrm{e}^{-\mathrm{t}} \mathrm{dt}=\gamma_{1}\left(\mathrm{a},-\log \left[1-\Phi\left(\frac{\mathrm{x}-\mu}{\sigma}\right)\right]\right)
$$

Evidently, the GN distribution is defined by a simple transformation: if $\mathrm{Z} \sim \mathrm{G}(\mathrm{a}, 1)$, then the random variable $\mathrm{X}=\Phi-1(1-\mathrm{e}-\mathrm{Z})$ has the density function (5). Hereafter, a random variable $\mathrm{X}$ following (5) is denoted by $X \sim G N(a, \mu, \sigma)$. The density function (5) does not involve any complicated function and the normal distribution arises as the basic exemplar for $\mathrm{a}=1$. It is a positive point of the current generalization. We motivate the paper by comparing the performances of the GN, normal and skewnormal models applied to two real data sets.

In Figure 1, we display some possible shapes of the density function (5) for some parameter values. It is evident that the GN distribution is much more flexible than the normal distribution.

The new distribution is easily simulated as follows: if $\mathrm{V}$ is a gamma random variable with parameter a, then

$$
X=\sigma \Phi^{-1}[1-\exp (-V)]+\mu
$$


has the $\operatorname{GN}(a, \mu, \sigma)$ distribution. This scheme is useful because of the existence of fast generators for gamma random variables and the standard normal quantile function is available in most statistical packages.

(a)

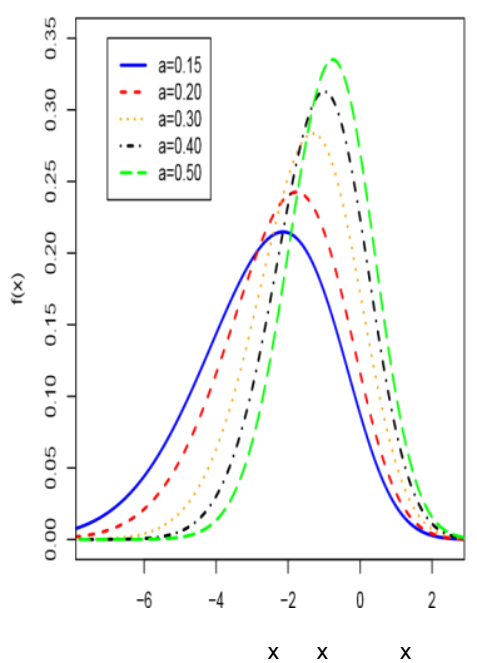

(b)

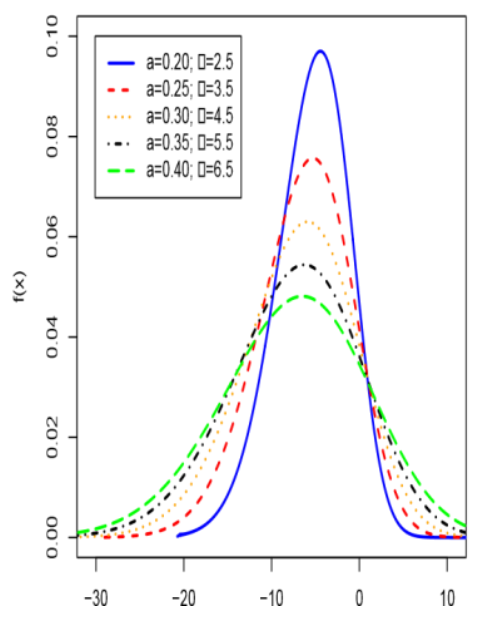

(c)

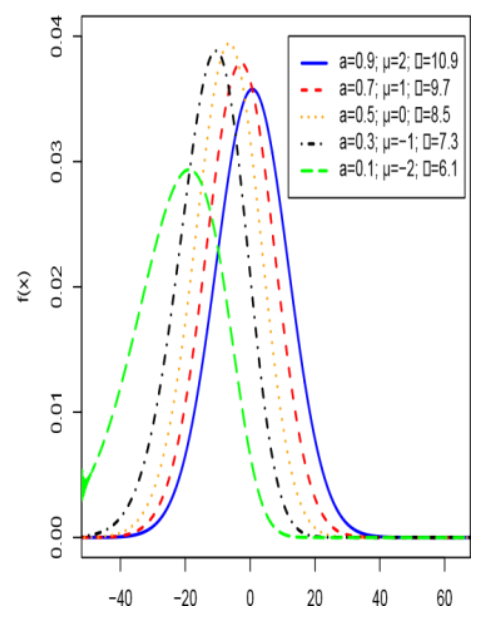

Figure 1: Plots of the new density function for some parameter values. (a) For different values of a with $\mu=0$ and $\sigma=1$. (b) For different values of a and $\sigma$ with $\mu=0$. (c) For different values of a, $\mu$ and $\sigma$.

\section{Useful expansions}

Expansions for equations (5) and (6) can be derived using the concept of exponentiated distributions. Consider the exponentiated normal (EN) distribution with power parameter a $>0$ defined by $\mathrm{Y} \sim \mathrm{EN}(\mathrm{a}, \mu, \sigma)$, with cdf and pdf given by $\mathrm{Ha}(\mathrm{y})=\Phi(\mathrm{y}-\sigma \mu) \mathrm{a}$ and $h_{a}(y)=\frac{a}{\sigma} \phi\left(\frac{y-\mu}{\sigma}\right) \Phi\left(\frac{y-\mu}{\sigma}\right)^{a-1}$, respectively.

The properties of several exponentiated distributions have been studied by some authors, see Mudholkar and Srivastava (1993) and Mudholkar et al. (1995) for exponentiated Weibull (EW), Gupta et al. (1998) for exponentiated Pareto, Gupta and Kundu (2001) for exponentiated exponential (EE) and Nadarajah and Gupta (2007) for exponentiated gamma (EG) distributions. More recently, Cordeiro et al. (2011) investigated these properties for the exponentiated generalized gamma (EGG) distribution.

Based on an expansion due to Nadarajah et al. (2013), we can write

$$
\left\{-\log \left[1-\Phi\left(\frac{y-\mu}{\sigma}\right)\right]\right\}^{a-1}=(a-1) \sum_{k=0}^{\infty}\left(\begin{array}{c}
k+1-a \\
k
\end{array}\right) \sum_{j=0}^{k} \frac{(-1)^{j+k}\left(\begin{array}{c}
k \\
j
\end{array}\right) p_{j, k}}{(a-1-j)} \Phi\left(\frac{y-\mu}{\sigma}\right)^{a+k-1}
$$

where $a>0$ is any real parameter and the constants $p_{j, k}$ can be calculated recursively by

$$
p_{j, k}=k^{-1} \sum_{m=1}^{k} \frac{(-1)^{m}[m(j+1)-k]}{(m+1)} p_{j, k-m^{\prime}}
$$

for $k=1,2, \ldots$ and $p_{j, 0}=1$. Let 


$$
b_{k}=\frac{\left(\begin{array}{c}
k+1-a \\
k
\end{array}\right)}{(a+k) \Gamma(a-1)} \sum_{j=0}^{k} \frac{(-1)^{j+k}\left(\begin{array}{c}
k \\
j
\end{array}\right) p_{j, k}}{(a-1-j)} .
$$

Then, equation (5) can be expressed as

$$
f(x)=\sum_{k=0}^{\infty} b_{k} h_{a+k}(x)
$$

where $h_{a+k}(x)=\left(\frac{a+k}{\sigma}\right) \phi\left(\frac{x-\mu}{\sigma}\right) \Phi\left(\frac{x-\mu}{\sigma}\right)^{a+k-1}$ denotes the $\operatorname{EN}(a+k, \mu, \sigma)$ density function. The cdf corresponding to (7) becomes

$$
F(x)=\sum_{k=0}^{\infty} b_{k} H_{a+k}(x)=\sum_{k=0}^{\infty} b_{k} \Phi\left(\frac{x-\mu}{\sigma}\right)^{a+k},
$$

where $H_{a+k}(x)=\Phi\left(\frac{x-\mu}{\sigma}\right)^{a+k}$ denotes the EN cdf with parameters $a+k, \mu$ and $\sigma$.

If $a>0$ is a real number, we can expand $\Phi\left(\frac{x-\mu}{\sigma}\right)^{a+k}$ as

$$
\Phi\left(\frac{x-\mu}{\sigma}\right)^{a+k}=\sum_{r=0}^{\infty} s_{r}(a+k) \Phi\left(\frac{x-\mu}{\sigma}\right)^{r},
$$

where

$$
s_{r}(a)=\sum_{l=r}^{\infty}(-1)^{r+l}\left(\begin{array}{l}
a \\
l
\end{array}\right)\left(\begin{array}{l}
l \\
r
\end{array}\right)
$$

Combining equations (8) and (9), we obtain

$$
F(x)=\sum_{k=0}^{\infty} \sum_{r=0}^{\infty} b_{k} s_{r}(a+k) \Phi\left(\frac{x-\mu}{\sigma}\right)^{r} .
$$

By differentiating the previous equation and changing indices, we can write

$$
f(x)=\sum_{r=0}^{\infty} d_{r} h_{r+1}(x)
$$

where $_{d_{r}}=\sum_{k=0}^{\infty} b_{k} s_{r+1}(a+k)$. Clearly, $\sum_{r=0}^{\infty} d_{r}=1$. Equation (11) is the main result of this section. It reveals that the GN density function is a linear combination of EN densities. So, several properties of the GN distribution can be obtained by knowing those properties of the EN distribution.

\section{Quantile Function}

The GN quantile function, say $\mathrm{Q}(\mathrm{u})=\mathrm{F}-1(\mathrm{u})$, can be expressed in terms of the normal quantile function $(\mathrm{QN}(\cdot))$. The normal quantile function is given by $\mathrm{x}=\mathrm{QN}(\mathrm{u})=\sigma \Phi-1(\mathrm{u})+\mu$. Inverting equation (6), we obtain the quantile function of $\mathrm{X}$ as

$$
F^{-1}(u)=Q_{G N}(u)=\mu+\sigma Q_{N}\left\{1-\exp \left[-Q^{-1}(a, 1-u)\right]\right\},
$$

for $0<\mathrm{u}<1$, where $\mathrm{Q}-1(\mathrm{a}, \mathrm{u})$ is the inverse function of $\mathrm{Q}(\mathrm{a}, \mathrm{z})=1-\gamma(\mathrm{a}, \mathrm{z}) / \Gamma(\mathrm{a})$. Quantities of interest can be obtained from (12) by substituting appropriate values for $\mathrm{u}$. Further, the normal quantile function can be expressed as (Steinbrecher, 2002) in equation (43), see Appendix A. Further, after some algebra (see Appendix A), we obtain 


$$
Q_{N}(u)=\sum_{s=0}^{\infty} w_{s} u^{s}
$$

where $w_{s}=\sum_{k=s}^{\infty}(-2)^{s-k}(\sqrt{2 \pi})^{k}\left(\begin{array}{l}k \\ s\end{array}\right) d_{k}$ and the quantity $d_{k}$ was defined in Section 3 .

We can obtain the inverse function $Q^{-1}(a, u)$ in the Wolfram website as

$$
z=Q^{-1}(a, 1-u)=\sum_{i=0}^{\infty} a_{i} u^{i / a},
$$

where $a_{0}=0, a_{1}=\Gamma(a+1)^{1 / a}, a_{2}=\Gamma(a+1)^{2 / a} /(a+1), a_{3}=(3 a+5) \Gamma(a+1)^{3 / a} /\left[2(a+1)^{2}(a+2)\right]$, etc.

We use throughout the paper an equation of Gradshteyn and Ryzhik (2007, Section 0.314) for a power series raised to a positive integer $j$ :

$$
\left(\sum_{i=0}^{\infty} a_{i} x^{i}\right)^{j}=\sum_{i=0}^{\infty} c_{j, i} x^{i}
$$

where the coefficients $c_{j, i}($ for $i=1,2, \ldots)$ are easily obtained from the recurrence equation

$$
c_{j, i}=\left(i a_{0}\right)^{-1} \sum_{m=1}^{i}[m(j+1)-i] a m c j, i-m
$$

$\operatorname{and}_{c_{j, 0}}=a_{0}^{j}$. The coefficient $c_{j, i}$ can be determined from $c_{j, 0}, \ldots, c_{j, i}-1$ and then from the quantities $a_{0}, \ldots, a_{i}$. In fact, $c_{j, i}$ can be given explicitly in terms of the coefficients $a_{i}$, although it is not necessary for programming numerically our expansions in any algebraic or numerical software.

By expanding the exponential function and using (14), we have (see Appendix A)

$$
1-\exp \left(-\sum_{r=0}^{\infty} a_{r} u^{r / a}\right)=1-\sum_{r=0}^{\infty} p_{r} u^{r / a},
$$

where the $p_{r}^{\prime}$ s are defined there. We can write

$$
Q_{G N}(u)=\mu+\sigma Q_{N}\left(1-\sum_{r=0}^{\infty} p_{r} u^{r / a}\right) .
$$

By using equations (13) and (14), we can obtain from (12)

$$
Q_{G N}(u)=\mu+\sigma \sum_{r=0}^{\infty} \bar{p}_{j} h_{j, r} u^{r / a},
$$

where $_{\bar{p}_{j}}=\sum_{s=0}^{\infty} \sum_{j=0}^{s}(-1)^{j} w_{s}\left(\begin{array}{l}s \\ j\end{array}\right){ }^{\text {and }} h_{j, i}=\left(i p_{0}\right)^{-1} \sum_{m=0}^{i}[m(j+1)-i] p_{m} h_{j, i-m} \quad$ Some algebraic details about (16) and others quantities of interest are given in Appendix A. Equations (13)-(15) are the main results of this section.

\section{Moments}

Here, we obtain the ordinary and incomplete moments of $X$. They can be immediately derived from the moments of $Y$ following the $\operatorname{EN}(a, \mu, \sigma)$ distribution. Hereafter, let $Z$ be the standard $\operatorname{GN}(a, 0,1)$ random variable. First, we obtain the moments of $Z$. Thus, we can write from (7)

$$
\mu_{n}^{\prime}=E\left(Z^{n}\right)=\sum_{k=0}^{\infty} b_{k} \int_{-\infty}^{\infty} x^{n} \Phi(x)^{a+k-1} \phi(x) d x .
$$


Further, we can express $\mu_{n}^{\prime}$ in terms of $Q_{N}(u)$ as

$$
\mu_{n}^{\prime}=\sum_{k=0}^{\infty} b_{k} \int_{0}^{1} Q_{N}(u)^{n} u^{a+k-1} d u .
$$

Using (13) and (14), we can rewrite $\mu_{n}^{\prime}$ as

$$
\mu_{n}^{\prime}=\sum_{k, s=0}^{\infty} \frac{b_{k} e_{n, s}}{(a+k+s)}
$$

Where the quantities $\mathrm{e}_{n, s}$ are determined from (13)-(15) as $\mathrm{e}_{n, s}=\left(i \omega_{0}\right)^{-1} \sum_{m=1}^{s}[m(n+1)-$ $s] \omega_{m} \mathrm{e}_{n, s-m}$ for $\mathrm{s} \geq 1, \mathrm{e}_{n, 0}=\omega_{0}^{n}, \omega_{m}=\sum_{k=m}^{\infty}(-2)^{m-k}(\sqrt{2 \pi})^{k}\left(\begin{array}{c}k \\ m\end{array}\right) d_{k}$ and the quanity $d_{k}$ was defined in Section 3.

The moments of $\mathrm{X}$ immediately follow from the moments of $\mathrm{Z}$ as $\mathrm{E}\left(\mathrm{X}^{n}\right)=\sum_{k=0}^{n}\left(\begin{array}{l}n \\ k\end{array}\right) \mu^{n-k} \sigma^{k} \mu_{k}^{\prime}$.

The second representation for $\mu^{\prime} \mathrm{n}$ is based on (n,r)th probability weighted moment (PWM) (for $n$ and $r$ positive integers) of the standard normal distribution given by

$$
\mu_{n}^{\prime}=\sum_{k, r=0}^{\infty} b_{k} s_{r+1}(a+k) \tau_{n, r}
$$

Where $\mathrm{s}_{r}(a)$ is given by (10) and $\tau_{n . r}$ can be expressed as (Nadarajah,2008)

$$
\begin{gathered}
\tau_{n, r}=2^{n / 2} \pi^{-(r+1 / 2)} \sum_{p=0}^{r}\left(\frac{\pi}{2}\right)^{p}\left(\begin{array}{l}
r \\
p
\end{array}\right) \Gamma\left(\frac{n+r-p+1}{2}\right) \times \\
F_{A}^{(r-p)}\left(\frac{n+r-p) \text { even }}{2} ; \frac{1}{2}, \ldots, \frac{1}{2} ; \frac{3}{2}, \ldots, \frac{3}{2} ;-1, \ldots,-1\right),
\end{gathered}
$$

where

$$
F_{A}^{(n)}\left(a, b_{1}, \ldots, b_{n} ; c_{1}, \ldots, c_{n} ; x_{1}, \ldots, c_{n}\right)=\sum_{m_{1}=0}^{\infty} \cdots \sum_{m_{n}=0}^{\infty} \frac{a_{m_{1}+\cdots+m_{n}}\left(b_{1}\right)_{m_{1}} \cdots\left(b_{n}\right)_{m_{n}}}{\left(c_{1}\right)_{m_{1}} \cdots\left(c_{n}\right)_{m_{n}}} \frac{x_{1}^{m_{1}} \cdots x_{n}^{m_{n}}}{m_{1} ! \cdots m_{n} !}
$$

is the Lauricella function of type A (Exton, 1978) and the Pochhammer symbol (a) $\mathrm{k}=$ $a(a+1) \ldots(a+k-1)$ indicates the kth rising factorial power of a with the convention $(a) 0=1$.

We derive three formulae for the $n$th incomplete moment of $\mathrm{Z}$ given by $\mathrm{E}(\mathrm{Zn} \mid \mathrm{Z}<\mathrm{y})=\operatorname{Tn}(\mathrm{y})=$ $\int_{0}^{y} x^{n} f(x) d x$. First, based on equation (11), with $\mu=0$ and $\sigma=1, \operatorname{Tn}(\mathrm{y})$ reduces to

$$
T_{n}(y)=\sum_{r=0}^{\infty} d_{r} \int_{-\infty}^{y} x^{n} \phi(x) \Phi(x)^{r} d x .
$$

We can write $\Phi(x)$ as a power series $\Phi(x)=\sum_{j=0}^{\infty} a_{j} x^{j}$, where $a_{0}=(1+\sqrt{2} / \pi)^{-1} / 2, a_{2 j+1}$

$=(-1)^{j} /\left[\sqrt{2} \pi 2^{j}(2 j+1) j !\right]$ for $j=0,1,2 \ldots$ and $a_{2 j}=0$ for $j=1,2, \ldots$ Further, using (14), we have

$$
\Phi(x)^{r}=\sum_{j=0}^{\infty} c_{r, j} x^{j}
$$

where the coefficients $c_{r, j}$ can be determined from the recurrence equation (15) with these $a_{i}^{\prime}$ s. Thus, using (21) and changing variable in the last integral, it follows from (20) 


$$
T_{n}(y)=\frac{1}{\sqrt{2 \pi}} \sum_{j, r=0}^{\infty} 2^{n+j-1} d_{r} c_{r, j} \gamma\left(\frac{n+j+1}{2}, \frac{y^{2}}{2}\right) .
$$

Next, we derive a second representation for the moments. The integral $A(j, q)=\int_{-\infty}^{q} x^{j} \mathrm{e}^{-\mathrm{x}^{2} / 2} \mathrm{dx}$ can be determined for $q>0$ and $q<0$. We define

$$
G(j)=\int_{0}^{\infty} x^{j} \mathrm{e}^{-\frac{\mathrm{x}^{2}}{2}} \mathrm{dx}=2^{(\mathrm{j}-1) / 2} \Gamma\left(\frac{\mathrm{j}+1}{2}\right) .
$$

For $q<0$ and $q>0$, we have

$$
A(j, q)=(-1)^{j} G(j)+(-1)^{j+1} H(j, q)
$$

and

$$
A(j, q)=(-1)^{j} G(j)+H(j, q)
$$

respectively, where the integral $H(j, q)=\int_{0}^{q} x^{j} \mathrm{e}^{-\mathrm{x}^{2} / 2} \mathrm{dx}$ can be easily computed (Whittaker and Watson, 1990). The details are given in Appendix B. After some algebra, we can write $T_{n}(y)$ as

$$
T_{n}(y)=\frac{1}{\sqrt{2 \pi}} \sum_{k, r, j=0}^{\infty} b_{k} c_{r, j} s_{r+1}(a+k) A(j+n, y)
$$

where ${ }_{c_{r, j}}=\left(j a_{0}\right)^{-1} \sum_{m=1}^{j}[m(r+1)-j] a_{m} c_{r, j-m}$, for $j \geq 1, c_{r, 0}=a_{0}^{r}, c_{0,0}=1, s_{r}(a)$ is given by (10) and the quantities $a_{i}^{\prime}$ are defined in Section 4. Some details about (23) are given in Appendix B.

A third representation for $T_{n}(y)$ is based on the normal quantile function. Thus, equation (21) becomes

$$
T_{n}(y)=\sum_{r=0}^{\infty} d_{r} \int_{0}^{\Phi(y)} Q_{N}(u)^{n} u^{r} d u .
$$

After some algebra, using (13) and (14), we have

$$
T_{n}(y)=\sum_{r, s=0}^{\infty} d_{r} e_{n, s} \frac{\Phi(y)^{s+r+1}}{(s+r+1)^{\prime}}
$$

where $e_{n, s}$ is given before. More details about (24) are addressed in Appendix B. The

$n$th incomplete moment of $\mathrm{X}$ follows after a binomial expansion

$$
E\left(X^{n} \mid X<y\right)=\sum_{k=0}^{n} \mu_{n-k}^{\prime} \sigma^{k}\left(\begin{array}{l}
n \\
k
\end{array}\right) T_{k}\left(\frac{y-\mu}{\sigma}\right)
$$

We can derive the mean deviations of $Z$ about the mean $\mu_{1}^{\prime}$ and about the median $M$ in terms of its first incomplete moment. They can be expressed as

$$
\delta_{1}=2\left[\mu_{1}^{\prime} F\left(\mu_{1}^{\prime}\right)-T_{1}\left(\mu_{1}^{\prime}\right)\right] \quad \delta_{2}=\mu_{1}^{\prime}-2 T_{1}(M)^{\prime}
$$

where $\mu_{1}^{\prime}=E(Z)$ and $T_{1}(q)=\int_{-\infty}^{q} x f(x) d x$. The quantity $T_{1}(q)$ can be obtained from (22) (or (23) or (24)) with $n=1$ and the measures $\delta_{1}$ and $\delta_{2}$ in (25) are immediately determined by setting $q=\mu_{1}^{\prime}$ and $q=$ $M$, respectively.

For a positive random variable $X$, the Bonferroni and Lorenz curves are defined by $B(\pi)=T_{1}(q) /\left(\pi \mu_{1}^{\prime}\right)$ 
and $L(\pi)=T_{1}(q) / \mu_{1}^{\prime}$, respectively, where $q=F^{-1}(\pi)=Q_{G N}(\pi)$ comes from the quantile function (12) for a given probability $\pi$.

Next, we obtain the probability weighted moments (PWMs) of $Z$. They cover the summarization and description of theoretical probability distributions. The primary use of these moments is to estimate the parameters of a distribution whose inverse cannot be expressed explicitly. The $(s, p)$ th PWM of $Z$ is formally defined as

$$
\xi_{s, p}=E\left[Z^{s} F(Z)^{p}\right]=\int_{0}^{\infty} z^{s} F(z)^{p} f(z) \mathrm{d} z .
$$

Using (8), (11) and (14), we obtain

$$
\xi_{s, p}=\frac{1}{\sqrt{\pi}} \sum_{j, n, r=0}^{\infty} 2^{\frac{(j+n+s)}{2}-1} d_{r} \bar{f}_{p, j} c_{r, n} \Gamma\left(\frac{j+n+s+1}{2}\right),
$$

where $d_{r}$ is definedin Section $3, \overline{\mathrm{f}}_{p, j}=\left(j e_{0}\right)^{-1} \sum_{v=1}^{j}[v(p+1)-j] e_{v} \bar{f}_{p, j-m}$ for $j \geq 1, \bar{f}_{p, 0}, e_{j}=\sum_{t=0}^{\infty} q_{t} c_{t, j} \quad$ and $q_{t}=\sum_{t=0}^{\infty} b_{t} s_{t}(a+k)$. The quantity $c_{t, j}$ was just defined after equation (23).

Equations (17)-(19), (22)-(24) and (26) are the main results of this section. Some algebraic details are given in Appendix B.

The skewness and kurtosis measures can be calculated from the ordinary moments using well- known relationships. Plots of the skewness and kurtosis for selected parameters values as function of $a$ are displayed in Figure 2. In the plots of Figures 2a and 2c, $\sigma=10.50$, whereas in those of Figures $2 \mathrm{~b}$ and $2 \mathrm{~d}, \mu=2.50$.

(a)

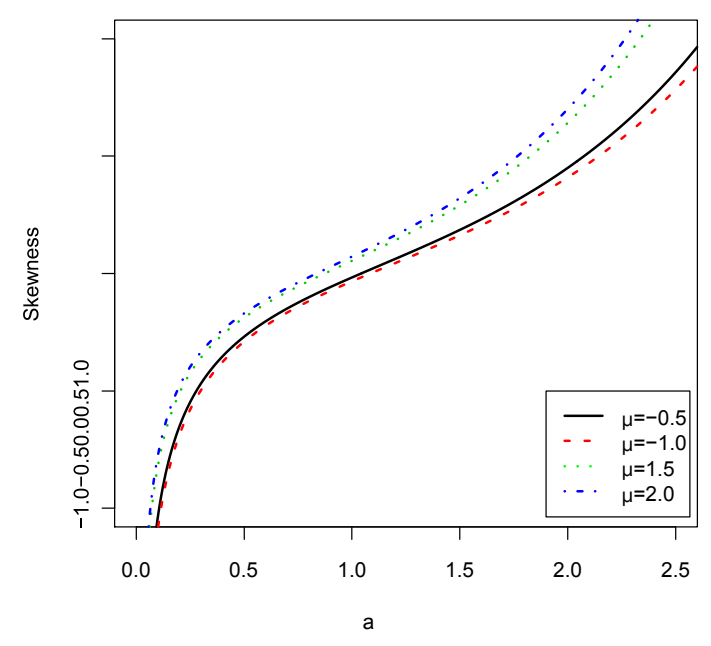

( b )

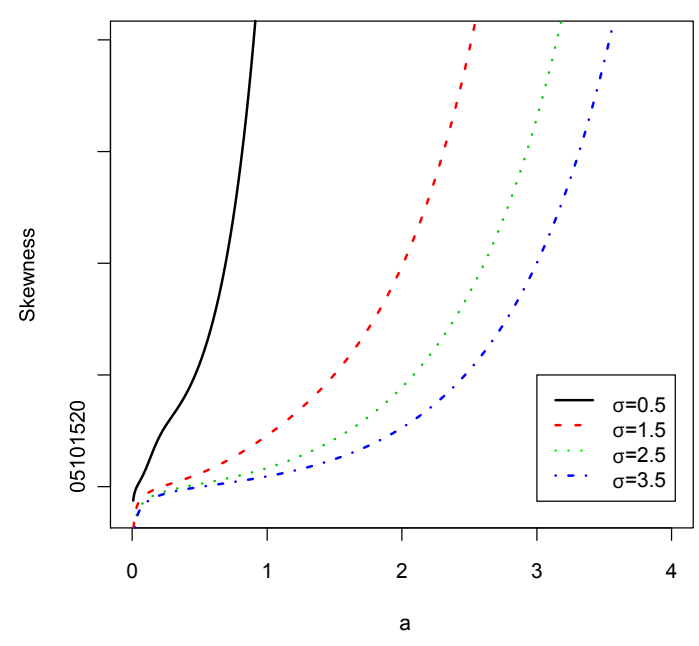


(c)

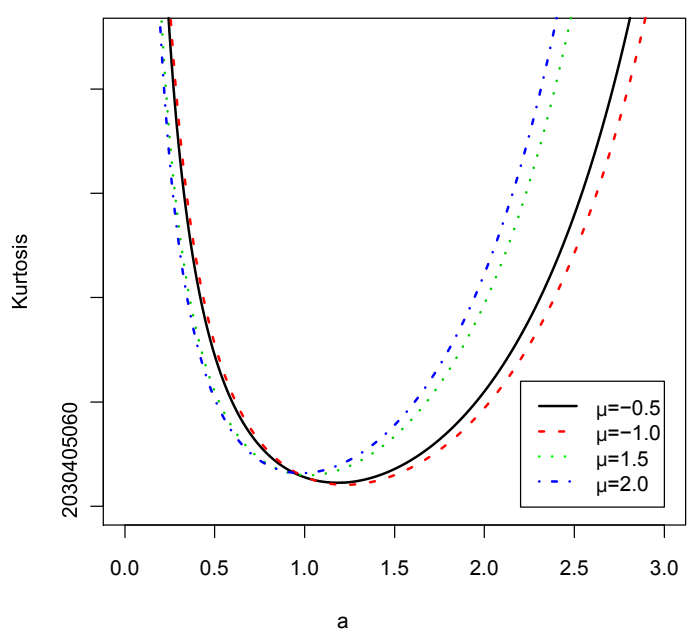

(d)

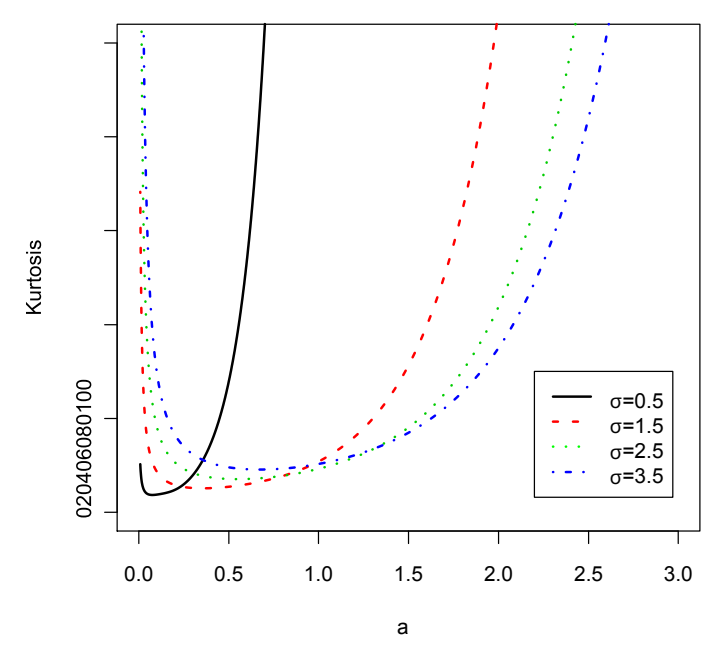

Figure 2: (a) Skewness of $X$ as function of $a$ for some values of $\mu$. (b) Skewness of $X$ as function of $a$ for some values of $\sigma$. (c) Kurtosis of $X$ as function of $a$ for some values of $\mu$. (d) Kurtosis of $X$ as function of $a$ for some values of $\sigma$.

\section{Generating function}

The generating function $M(-t)=E\left(\mathrm{e}^{-\mathrm{tZ}}\right)$ of $Z \sim \mathrm{GN}(a, 0,1)$ is given by

$$
M(-t)=\frac{1}{\sqrt{2} \pi} \sum_{k, r=0}^{\infty} b_{k} s_{r+1}(a+k) \int_{-\infty}^{\infty} \Phi(x)^{r} \exp \left(-t x-\frac{x^{2}}{2}\right) d x .
$$

Inserting equation (21), we obtain

$$
M(-t)=\frac{1}{\sqrt{2} \pi} \sum_{k, r, j=0}^{\infty} b_{k} s_{r+1}(a+k) c_{r, j} \int_{-\infty}^{\infty} x^{j} \exp \left(-t x-\frac{x^{2}}{2}\right) d x .
$$

Based on Prudnikov et al. (1986,Eq.2.3.15.8), the above integral can be rewritten as

$$
J(s, j)=\int_{-\infty}^{\infty} x^{j} \exp \left(-s x-\frac{x^{2}}{2}\right) d x=(-1)^{j} \sqrt{2 \pi} \frac{\partial^{j}}{\partial s^{j}}\left(\mathrm{e}^{\mathrm{s}^{2} / 2}\right) .
$$

Thus, the moment generating function (mgf) of $\mathrm{Z}$ becomes

$$
M(-t)=\frac{1}{\sqrt{2} \pi} \sum_{k, r, j=0}^{\infty} b_{k} s_{r+1}(a+k) c_{r, j} J(s, j)
$$

A second representation for $M(t)$ can be based on the quantile function. We have

$$
M(t)=\int_{0}^{1} \exp \left[t Q_{G N}(u)\right] d u .
$$

Expanding the exponential function, using (16) and after some algebra, we obtain 


$$
M(t)=\sum_{k, r=0}^{\infty} \frac{d_{k, r}}{\left(\frac{r}{a}+1\right)} \frac{t^{k}}{k !}
$$

where $d_{k, r}=\left(r g_{0}\right)^{-1} \sum_{m=1}^{r}[m(k+1)-r] g_{m} d_{k, r-m}$ for $r \geq 1, d_{k, 0}=g_{0}^{k}, d_{0, r}=1, g_{j}=\bar{p}_{j} h_{j, r}$ and the quantities $p_{j}$ and $h_{j, r}$ are given in Section 4 .

Equations (27) and (28) are the main results of this section. The mgf of $X$ is simply given by $\operatorname{MX}(\mathrm{t})=\mathrm{e} \mu \mathrm{M}(\sigma \mathrm{t})$, where $1=\sqrt{ }-1$. The characteristic function (cf) has many useful and important properties which gives it a central role in statistical theory. Its approach is particularly useful in analysis of linear combination of independent random variables. Clearly, a simple representation for the characteristic

function $(\mathrm{chf}) \phi_{X}(t)=M_{X}(\mathrm{it})$ of $X$, where $\mathrm{i}=\sqrt{ }-1$, is given by

$$
\phi_{X}(t)=\int_{0}^{\infty} \cos (t x) f(x) d x+\mathrm{i} \int_{0}^{\infty} \sin (\mathrm{tx}) \mathrm{f}(\mathrm{x}) \mathrm{dx} .
$$

From the expansions $\cos (t x)=\sum_{r=0}^{\infty} \frac{(-1)^{r}}{(2 r) !}(t x)^{2 r}$ and $\sin (t x)=\sum_{r=0}^{\infty} \frac{(-1)^{r}}{(2 r+1) !}(t x)^{2 r+1}$, we obgtain

$$
\phi_{x}(t)=\sum_{r=0}^{\infty} \frac{(-1)^{r} t^{2 r}}{(2 r) !} E\left(X^{2 r}\right)+\mathrm{i} \sum_{\mathrm{r}=0}^{\infty} \frac{(-1)^{\mathrm{r}} \mathrm{t}^{2 \mathrm{r}+1}}{(2 \mathrm{r}+1) !} \mathrm{E}(\mathrm{X} 2 \mathrm{r}+1)
$$

\section{Entropies}

An entropy is a measure of variation or uncertainty of a random variable X. Two popular entropy measures are the Rényi and Shannon entropies (Shannon, 1951; Rényi, 1961). Here we consider therandom variable $Z \sim G N(a, 0,1)$. Thus, the Rényi entropy is defined as

for $\gamma>0$ and $\gamma \neq 1$.

$$
I_{R}(\gamma)=\frac{1}{1-\gamma} \log \int_{-\infty}^{+\infty} f^{\gamma}(x) d x
$$

First, we consider $\gamma=\mathrm{n}=2,3, \ldots, \mu=0, \sigma=1$ and the rth moment of the standard normal distribution given by

$$
m_{r}^{\prime}=\frac{1}{\sqrt{2 \pi}} \int_{-\infty}^{+\infty} x^{r} \mathrm{e}^{-\mathrm{x}^{2} / 2}
$$

We have two cases: $m^{\prime} r=0$, if $r$ is odd, and $m^{\prime} r=1 \times 3 \ldots \times(r-1)$, if $r$ is even.

Using (21), we can write from (11) and (21)

$$
\begin{aligned}
I_{R}(n) & =\frac{1}{1-n} \log \left\{\left(\frac{1}{\sqrt{2 \pi}}\right)^{n} \sum_{j=0}^{n} \wp_{n, j} \int_{-\infty}^{+\infty} x^{j} \mathrm{e}^{-\frac{n x^{2}}{2}} \mathrm{dx}\right\} \\
& =\frac{1}{1-n}\left\{-\frac{(n-1)}{2} \log (2 \pi)-\frac{(j+1)}{2} \log (n)+\log \left[\sum_{j=0}^{n} \wp_{n, j} m_{j}^{\prime}\right]\right\}
\end{aligned}
$$

Where $\wp_{n, j}=\left(j e_{0}\right)^{-1} \sum_{m=1}^{j}[m(n+1)-j] e_{m} \wp_{n, j-m}, e_{j}=\sum_{j=0}^{\infty} d_{r} c_{r, j}, \wp_{n, 0}=e_{0}^{n}$ and the $\mathrm{m}^{\prime} \mathrm{j}$ 's are given by (29). The quantities dr's are defined in Section 3, whereas the cr,j's and the av's are given in Section 
5.

We can write $I_{R}(\gamma)=(1-\gamma)^{-1} E\left\{f(Z)^{\gamma-1}\right\}$. Let $\delta=E(Z)$. For $\gamma$ real positive, we have

$$
E\left\{f(Z)^{\gamma-1}\right\}=\delta^{\gamma-1} E\{1+\theta[f(Z)-\delta]\}^{\gamma-1},
$$

where $\theta=\delta^{-1}$. From the generalized binomial expansion, we obtain

where $_{\Im_{n}}=\prod_{j=0}^{n-1}(\gamma-1-j)$. Further,

$$
\{1+\theta[f(Z)-\delta]\}^{\gamma-1}=1+\sum_{n=1}^{\infty} \frac{\theta^{n} \Im_{n}}{n !}[f(Z)-\delta]^{n},
$$

$$
E\left\{f(Z)^{\gamma-1}\right\}=\delta^{\gamma-1}\left(1+\sum_{n=2}^{\infty} \frac{\theta^{n} \Im_{n}}{n !} E\left\{[f(Z)-\delta]^{n}\right\}\right)
$$

We now obtain $E\{[f(Z)] n\}$ for $n \geq 2$. From equation (11) and using the binomial expansion, we can write

$$
\rho_{n}=E\left\{[f(Z)]^{n}\right\}=\sum_{j=0}^{\infty} \wp_{n, j} \psi_{n, j^{\prime}}
$$

where $\psi_{n, j}=E\left\{Z^{j} \phi(Z)^{n}\right\}$. Thus,

$$
\psi_{n, j}=\int_{-\infty}^{\infty} x^{j} \phi(x)^{n+1} \mathrm{dx}
$$

Setting $\sqrt{(n+1) x}=y$, we can easily determine the last integral and then rewrite $\rho_{n}$ as

$$
\rho_{n}=\left(\frac{1}{\sqrt{2 \pi}}\right)^{n} \sum_{j=0}^{\infty} \wp_{n, j}\left(\frac{1}{\sqrt{n+1}}\right)^{j+1} m_{j}^{\prime}
$$

By expanding the binomial term in (31), we can obtain an explicit expression for $I_{R}(\gamma)$, which holds for any $\gamma$ real positive and $\gamma=1$, given by

$$
I_{R}(\gamma)=(1-\gamma)^{-1} \delta^{\gamma-1}\left[1+\sum_{n=2}^{\infty} \frac{\theta^{n} \Im_{n}}{n !} \sum_{k=0}^{n}(-\delta)^{n-k}\left(\begin{array}{l}
n \\
k
\end{array}\right) \rho_{k}\right],
$$

where $\rho \mathrm{k}$ is determined from (32). Algebraic details can be found in Appendix D.

Next, the Shannon entropy of a random variable $\mathrm{Z}$ is defined by $\mathrm{E}\{-\log [\mathrm{f}(\mathrm{Z})]\}$. It is a special case of the Rényi entropy when $\gamma \uparrow 1$. Equation (30) is very complicated for limiting, and then we derive an explicit expression for the Shannon entropy from its definition. We can write

$$
\begin{aligned}
\log [f(x)] & =\log \left\{\frac{1}{\sigma \Gamma(a)} \phi(x)\{-\log [1-\Phi(x)]\}^{a-1}\right\} \\
& =-\log [\sigma \Gamma(a)]+\log [\phi(x)]+(a-1) \log \{-\log [1-\Phi(x)]\}
\end{aligned}
$$

So, we first calculate $\mathrm{E}\{\log [\phi(\mathrm{X})]\}$ and $\mathrm{E}[\log \{-\log [1-\Phi(\mathrm{X})]\}]$. Setting $\mu=0$ and $\sigma=1$, the first quantity is easily calculated as follows

$$
E\{\log [\phi(X)]\}=-\frac{1}{2} \log (2 \pi)-E\left(\frac{X^{2}}{2}\right)=-\frac{1}{2}\left[\log (2 \pi)+\mu_{2}^{\prime}\right\}
$$

where $\mu_{2}^{\prime}$ comes from (17) or (18) with $\mathrm{n}=2$. 
The second quantity $\mathrm{E}[\log \{-\log [1-\Phi(\mathrm{x})]\}]$ is obtained from the expansion of $\log \{-\log [1-$ $\Phi(\mathrm{x})]\}$. We can write (for $0<\mathrm{u}<1$ ) from MATHEMATICA

$$
\begin{aligned}
\log \{-\log [1-u]\} & =\log (u)+\frac{u}{2}+\frac{5 u^{2}}{24}+\frac{u^{3}}{8}+\frac{251 u^{4}}{2880}+\frac{19 u^{5}}{288}+\frac{19087 u^{6}}{362880}+\frac{751 u^{7}}{17280}+\frac{1070017 u^{8}}{29030400} \\
& =+\frac{2857 u^{9}}{89600}+\frac{26842253 u^{10}}{958003200}+O\left(u^{11}\right) .
\end{aligned}
$$

From equations (34)-(36), we obtain the Shanon entropy $\mathrm{E}\{-\log [\mathrm{f}(\mathrm{Z})]\}$ using the ordinary moments given by (17), (18) and (21). Equations (30), (33)-(36) are the main results of this section.

\section{Order statistics}

Order statistics have been used in a wide range of problems, including robust statistical estimation and detection of outliers, characterization of probability distributions and goodness-of-fit tests, entropy estimation, analysis of censored samples, reliability analysis, quality control and strength of materials.

Suppose $\mathrm{Z1}, \ldots, \mathrm{Zn}$ is a random sample from the standard GN distribution and let $\mathrm{Z1}: \mathrm{n}<\ldots<\mathrm{Zi}: \mathrm{n}$ denote the corresponding order statistics. Using (7) and (8), the pdf of Zi:n can be expressed as

$$
\begin{aligned}
f_{i: n}(z) & =\frac{n !}{(i-1) !(n-i) !} \sum_{j=0}^{n-i}(-1)^{j}\left(\begin{array}{c}
n-i \\
j
\end{array}\right) f(z) F(z)^{i+j-1} \\
& =\frac{n !}{(i-1) !(n-i) !} \sum_{j=0}^{n-i}(-1)^{j}\left(\begin{array}{c}
n-i \\
j
\end{array}\right)\left[\sum_{r=0}^{\infty} b_{r}(a+r) \Phi(z)^{a+r-1} \phi(z)\right]\left[\sum_{k=0}^{\infty} b_{k} \Phi(z)^{a+k}\right]^{i+j-1} .
\end{aligned}
$$

Based on equations (14) and (15), we obtain

$$
\left[\sum_{k=0}^{\infty} b_{k} \Phi(z)^{a+k}\right]^{i+j-1}=\sum_{k=0}^{\infty} \eta_{i+j-1, k} \Phi(z)^{(i+j-1) a+k},
$$

where $n_{i+j-1,0}=b_{0}^{i+j-1}$ and $n_{i+j-1, k}=\left(k b_{0}\right)^{-1} \sum_{m=1}^{k}[m(i+j)-k] b_{m} n_{i+j-1, k-m}$. Hence, the pdf of $Z_{i: n}$ reduces to

$$
f_{i: n}(z)=\phi(z) \sum_{j=0}^{n-i} \sum_{k=0}^{\infty} m_{j, k, r} \Phi(z)^{(i+j) a+k+r-1}
$$

where

$$
m_{j, k, r}=\frac{(-1)^{j}(a+r) n ! b_{r} \eta_{i+j-1, k}}{(i-1) !(n-i-j) ! j !}
$$

Equation (37) can be expressed as

$$
f_{i: n}(z)=\sum_{j=0}^{n-i} \sum_{k=0}^{\infty} f_{j, k, r} h_{(i+j) a+k+r}(z),
$$

where

$$
f_{j, k, r}=\frac{m_{j, k, r}}{[(i+j) a+k+r]} .
$$

Equation (38) is the main result of this section. It reveals that the pdf of the standard GN order statistics is a triple linear combination of $\mathrm{EN}$ densities with parameters $(\mathrm{i}+\mathrm{j}) \mathrm{a}+\mathrm{k}+\mathrm{r}, \mu=0$ and $\sigma=1$. So, several mathematical quantities of the GN order statistics such as ordinary and incomplete moments, mgf and mean deviations can be immediately obtained from those quantities of the EN 
distribution. It gives the density function of the GN order statistics as a power series of the standard normal cumulative function multiplied by the standard normal density function.

As an application of (37), the sth ordinary moment of Zi:n becomes

$$
E\left(Z_{i: n}^{s}\right)=\sum_{j=0}^{n-i} \sum_{r, k=0}^{\infty} m_{j, k, r} \tau_{s,(i+j) a+k+r-1}
$$

where $\tau \mathrm{s},(\mathrm{i}+\mathrm{j}) \mathrm{a}+\mathrm{k}+\mathrm{r}-1$ can be obtained from (19).

Another closed-form expression for $E\left(Z_{i: n}^{s}\right)$ can be derived using a result due to Barakat and Abdelkader (2004) applied to the independent and identically distributed case. Thus,

$$
E\left(Z_{i: n}^{s}\right)=s \sum_{j=n-i+1}^{n}(-1)^{i+j-n-1}\left(\begin{array}{l}
j-1 \\
n-i
\end{array}\right)\left(\begin{array}{l}
n \\
j
\end{array}\right) J_{j}(s)^{\prime}
$$

where $J_{j}(s)=\int_{0}^{\infty} z^{s-1}[1-F(z)]^{j} d x$. By expanding $[1-F(z)]^{j}$ and using (8), we obtain $J_{j}(s)$. For any real $a>0$, we can write from equations (8) and (15)

$$
\begin{aligned}
J_{j}(s) & =\sum_{m=0}^{j}(-1)^{m}\left(\begin{array}{c}
j \\
m
\end{array}\right) \int_{0}^{\infty} z^{s-1}\left(\sum_{k=0}^{\infty} b_{k} \Phi(z)^{a+k}\right)^{m} d x \\
& =\sum_{m=0}^{j}(-1)^{m}\left(\begin{array}{c}
j \\
m
\end{array}\right) \sum_{k=0}^{\infty} d_{m, k} \tau_{s-1, m a+k}=\sum_{k=0}^{\infty} \sum_{m=0}^{j}(-1)^{m}\left(\begin{array}{c}
j \\
m
\end{array}\right) d_{m, k} \tau_{s-1, m a+k},
\end{aligned}
$$

where $d_{m, k}$ is defined in Section 6 and the quantities $\tau_{n, r}$ are given in equation (19).

\section{Estimation}

Here, we consider estimation of the unknown parameters of the GL distribution by the method of maximum likelihood. Let $x 1, \ldots, x n$ be a random sample of size $n$ from the $G N(a, \mu, \sigma)$ distribution. The $\log$-likelihood function for the vector of parameters $\theta=(a, \mu, \sigma) \mathrm{T}$ can be expressed as

$$
\begin{aligned}
l(\boldsymbol{\theta})= & -n \log (\sigma)-n \log [\Gamma(a)]+\sum_{i=1}^{n} \log \left[\phi\left(\frac{x_{i}-\mu}{\sigma}\right)\right] \\
& +(a-1) \sum_{i=1}^{n} \log \left\{-\log \left[1-\Phi\left(\frac{x_{i}-\mu}{\sigma}\right)\right]\right\} .
\end{aligned}
$$

The components of the score vector $U(\theta)$ are given by

$$
\begin{aligned}
& U_{a}(\boldsymbol{\theta})=-n \psi(a)+\sum_{i=1}^{n} \log \left\{-\log \left[1-\Phi\left(\frac{x_{i}-\mu}{\sigma}\right)\right]\right\}, \\
& U_{\mu}(\boldsymbol{\theta})=\frac{1}{\sigma}+\sum_{i=1}^{n}\left(\frac{x_{i}-\mu}{\sigma}\right)+\frac{(a-1)}{\sigma} \sum_{i=1}^{n} \frac{\phi\left(\frac{x_{i}-\mu}{\sigma}\right)}{\left[1-\Phi\left(\frac{x_{i}-\mu}{\sigma}\right)\right] \log \left[1-\Phi\left(\frac{x_{i}-\mu}{\sigma}\right)\right]}, \\
& U_{\sigma}(\boldsymbol{\theta})=-\frac{n}{\sigma}+\frac{1}{\sigma} \sum_{i=1}^{n}\left(\frac{x_{i}-\mu}{\sigma}\right)^{2} \frac{(a-1)}{\sigma} \sum_{i=1}^{n} \frac{\left(\frac{x_{i}-\mu}{\sigma}\right) \phi\left(\frac{x_{i}-\mu}{\sigma}\right)}{\left[1-\Phi\left(\frac{x_{i}-\mu}{\sigma}\right)\right] \log \left[1-\Phi\left(\frac{x_{i}-\mu}{\sigma}\right)\right]},
\end{aligned}
$$

where $\psi(\cdot)$ is the digamma function.

Setting these expressions to zero and solving them simultaneously yields the maximum likelihood estimates (MLEs) of the three parameters. We use the matrix programming language Ox ( MaxBFGS subroutine), see for example, Doornik (2006) and the procedure NLMixed in SAS to compute the 
MLE $\theta$. For interval estimation of the model parameters, we require the expected information matrix. The $3 b \times 3$ total observed information matrix $\mathrm{J}(\theta)$ is given by

$$
J(\boldsymbol{\theta})=\left(\begin{array}{ccc}
J_{a a} & J_{a \mu} & J_{a \sigma} \\
\cdot & J_{\mu \mu} & J_{\mu \sigma} \\
\cdot & \cdot & J_{\sigma \sigma}
\end{array}\right),
$$

whose elements are listed in Appendix E. Under conditions that are fulfilled for parameters in the interior of the parameter space but not on the boundary, the asymptotic distribution of $\sqrt{ } n(\theta b-\theta)$ is $\mathrm{N} 3(0, \mathrm{~K}(\theta)-1)$, where $\mathrm{K}(\theta)=\mathrm{E}\{\mathrm{J}(\theta)\}$ is the expected information matrix. The multivariate normal $\mathrm{N} 3(0, \mathrm{~J}(\theta)-1)$ distribution can be used to construct approximate confidence intervals for the parameters.

The likelihood ratio (LR) can be used for testing the goodness of fit of the GL distribution and for comparing this distribution with the normal model. We can compute the maximum values of the unrestricted and restricted log-likelihoods to construct LR statistics for testing some sub-models of the GL distribution. For example, we may use the LR statistic to check if the fit using the new distribution is statistically "superior" to a fit using the normal distribution for a given data set. In any case, hypothesis tests of the type $\mathrm{H} 0: \psi=\psi 0$ versus $\mathrm{H}: \psi \neq \neq \psi$, where $\psi$ is a vector formed with some components of $\theta$ and $\psi 0$ is a specified vector, can be performed using LR statistics. For example, the test of $\mathrm{HO}: \mathrm{a}=1$ versus $\mathrm{H}: \mathrm{HO}$ is not true is equivalent to compare the $\mathrm{GN}$ and normal distributions and then the LR statistic reduces to $\mathrm{w}=2\{\ell(\mathrm{a}, \mu, \sigma)-\ell(1, \mu, \sigma)\}$, where $\mathrm{a}, \mu$ and $\sigma$ are the MLEs under $\mathrm{H}$ and $\mu$ and $\sigma$ are the estimates under $\mathrm{H} 0$.

\section{Applications}

In this section, the potentiality of the GN model is illustrated in two applications to real data. An alternative analysis of these data can be performed using the normal distribution. The beta-normal ( BN ) (Eugene et al., 2002) and Kumaraswamy-normal (KwN) models extend the normal model and they can also used to fit data that come from a distribution with heavy tails reducing the influence of aberrant observations.

\section{The BN distribution}

The $\mathrm{BN}$ density function with parameters $\mu$ and $\sigma$ and two extra shape parameters $\alpha>0$ and $\beta>0$ is given by

$$
f(x)=\frac{\Gamma(\alpha+\beta)}{\sigma \Gamma(\alpha) \Gamma(\beta)}\left[\Phi\left(\frac{x-\mu}{\sigma}\right)\right]^{\alpha-1}\left[1-\Phi\left(\frac{y-\mu}{\sigma}\right)\right]^{\beta-1} \phi\left(\frac{x-\mu}{\sigma}\right), \quad-\infty<x<\infty,
$$

For $\alpha=\beta=1$, we obtain the normal distribution. Recently, Alexander et al. (2012) and Cordeiro et al. (2012) proposed the generalized beta-generated and McDonald normal distributions, respectively. The first generated model contains, as special cases, several important distributions discussed in the literature such as the normal, exponentiated normal, $\mathrm{BN}$ and $\mathrm{KwN}$ distributions, among others.

\section{Kumaraswamy-normal (KwN) distribution}

The KwN density function with parameters $\mu$ and $\sigma$ and two extra shape parameters $a>0$ and $b>0$ is given by

$$
f(x)=\frac{a b}{\sigma} \phi\left(\frac{x-\mu}{\sigma}\right)\left[\Phi\left(\frac{x-\mu}{\sigma}\right)\right]^{a-1}\left[1-\Phi^{a}\left(\frac{x-\mu}{\sigma}\right)\right]^{b-1}, \quad-\infty<x<\infty .
$$

For $a=b=1$, we have the normal distribution. Clearly, equation (41) is much simpler than (40). 


\subsection{Application 1: Carbohydrates data}

The first example refers to the data from on agronomic experiments (Matsuo, 1986) conducted at the Federal University of Paraná. The main objective was to verify the content of carbohydrates (in \%) of the corn farms. Some summary statistics for the CO data are: mean=66.34, median=66.64, minimum $=62.35$ and maximum $=68.46$.

The parameters of each model are estimated by maximum likelihood (Section 9) using the subroutine NLMixed in SAS. We report the MLEs (and the corresponding standard errors in parentheses) of the parameters and the values of the Akaike Information Criterion (AIC), Consistent Akaike Information Criterion (CAIC) and Bayesian Information Criterion (BIC) in Table 2. The lower the values of these criteria, the better the fit. Since the values of these statistics are smaller for the GN distribution compared to their values for the other three models, we can conclude that the new distribution is the best model among the four to explain the current data. An analysis under the GN model also provides a check on the appropriateness of the normal model and indicates the extent for which inferences depend upon the model. For example, the LR statistic for testing the hypothesis H0 : $\mathrm{a}=1$ versus $\mathrm{H}: \mathrm{H} 0$ is not true, i.e. to compare the $\mathrm{GN}$ and normal models, is $\mathrm{w}=2\{-63.05-(65.20)\}$ $=4.30($ p-value $=0.0381)$, which provides support toward to the new model.

Table 1: MLEs and information criteria.

\begin{tabular}{c|cccc|ccc}
\hline \hline Carbon monoxide & & $a$ & $\mu$ & $\sigma$ & AIC & CAIC & BIC \\
\hline GN & & 0.1454 & 68.3276 & 0.7443 & 132.1 & 132.9 & 136.9 \\
& & $(0.0277)$ & $(0.2963)$ & $(0.0388)$ & & & \\
\hline Normal & & 1 & 66.3379 & 1.4800 & 134.3 & 134.8 & 137.6 \\
& & - & $(0.2467)$ & $(0.1744)$ & & & \\
\hline & $\alpha$ & $\beta$ & $\mu$ & $\sigma$ & & & \\
\hline BN & 0.1167 & 0.0678 & 65.5745 & 0.3683 & 137.3 & 138.6 & 143.6 \\
& $(0.0471)$ & $(0.0129)$ & $(0.3649)$ & $(0.0475)$ & & & \\
& $a$ & $b$ & $\mu$ & $\sigma$ & & & \\
\hline KwN & 0.1859 & 0.0309 & 66.6857 & 0.3460 & 132.2 & 133.3 & 138.3 \\
& $(0.2023)$ & $(0.0281)$ & $(1.1912)$ & $(0.1034)$ & & & \\
\hline \hline
\end{tabular}

Figure 3 displays the estimated densities and cumulative functions and the empirical cdf for the GN and normal models. These plots reveal a better GN fit to these data.

\subsection{Application 2: Carbon monoxide data}

Here, we work with carbon monoxide (CO) measurements made in several brands of cigarettes in 1998. The data have been collected by the Federal Trade Commission (FTC), an independent agency of the United States government, whose main mission is the promotion of consumer protection. For three decades the FTC regularly has released reports on the nicotine and tar content of cigarettes. The reports indicate that nicotine levels, on average, had remained stable since 1980, after falling in the preceding decade. The report entitled "Tar, Nicotine, and Carbon Monoxide of the Smoke of 1206 Varieties of Domestic Cigarettes for the year of 1998" at http://www.ftc.gov/reports/tobacco includes the data sets and some information about the source of the data, smoker's behavior and beliefs about nicotine, tar and carbon monoxide contents in cigarettes.

The CO data set can be found at http://home.att.net/ rdavis2/cigra.html. The data include $\mathrm{n}=384$ records of $\mathrm{CO}$ measurements, in milligrams, in cigarettes of several brands. Some summary statistics for the $\mathrm{CO}$ data are: mean=11.34, median=12.00, minimum $=0.05$ and maximum $=22.00$. In each case, the parameters are estimated by maximum likelihood using the subroutine NLMixed in SAS. We 
report the MLEs (and the corresponding standard errors in parentheses) of the parameters and the values of the AIC, CAIC and BIC statistics in Table 2. Since the values of these statistics are smaller for the GN and $\mathrm{KwN}$ distributions compared to those values for the other models, the new distribution

(a)

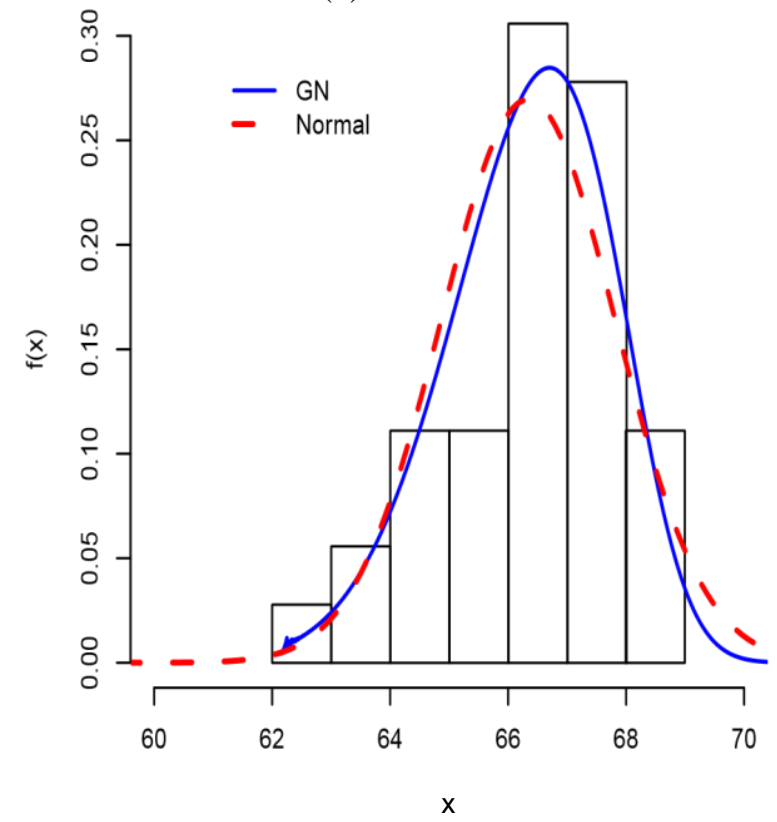

( b )

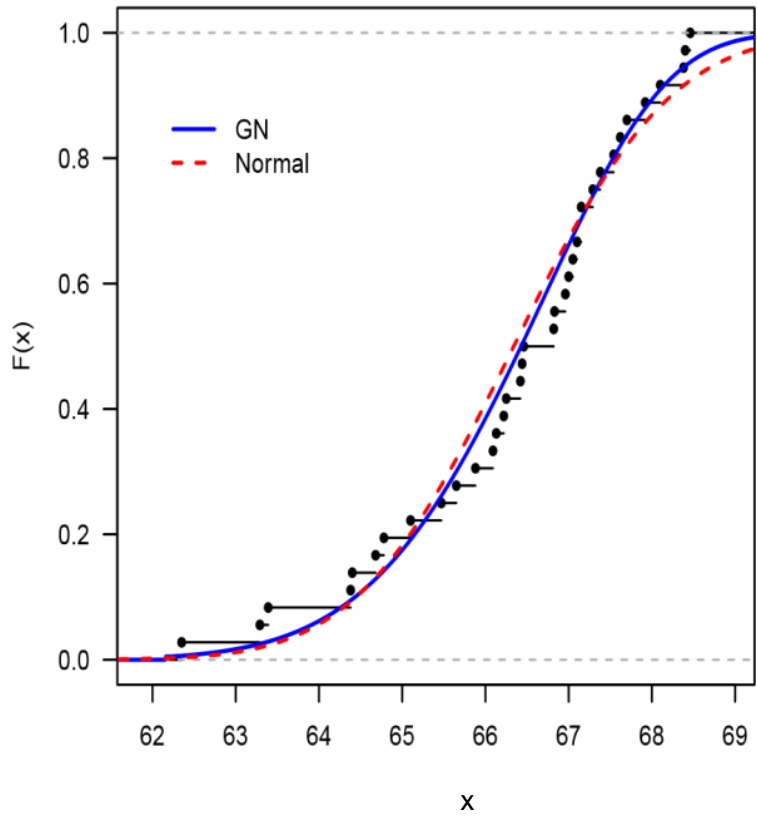

Figure 3: (a) Estimated densities of the GN and normal models for carbohydrates data. (b) Estimated cumulative functions and the empirical cdf for Carbohydrates data.

is a very competitive model to explain these data and it is more parsimonious. The LR statistic for comparing the GN and normal models is $w=2\{-962.9-(-1946.4)\}=20.6(\mathrm{p}$-value $=<0.0001)$, which yields favorable support toward to the first model.

Table 2: MLEs and information criteria.

\begin{tabular}{c|cccc|ccc}
\hline \hline Carbon monoxide & & $a$ & $\mu$ & $\sigma$ & AIC & CAIC & BIC \\
\hline GN & & 0.1432 & 16.9819 & 2.0889 & 1931.8 & 1931.9 & 1943.3 \\
& & $(0.0085)$ & $(0.2476)$ & $(0.0378)$ & & & \\
\hline Normal & & 1 & 11.3425 & 4.0626 & 1950.4 & 1950.5 & 1958.1 \\
& & - & $(0.2187)$ & $(0.1547)$ & & & \\
\hline & $\alpha$ & $\beta$ & $\mu$ & $\sigma$ & & & \\
\hline BN & 0.2143 & 3.1422 & 18.5092 & 2.8673 & 1932.9 & 1933.0 & 1948.3 \\
& $(0.0906)$ & $(0.4851)$ & $(0.4680)$ & $(0.5866)$ & & & \\
\hline & $a$ & $b$ & $\mu$ & $\sigma$ & & & \\
\hline KwN & 0.2242 & 0.0730 & 11.8209 & 1.2921 & 1929.1 & 1929.2 & 1944.5 \\
& $(0.0420)$ & $(0.0262)$ & $(1.1516)$ & $(0.1306)$ & & & \\
\hline
\end{tabular}

Figure 4 displays the estimated densities and estimated cumulative functions and the empirical cdf for the BN and normal models. So, the proposed model provides a better fit to these data. 
(a)

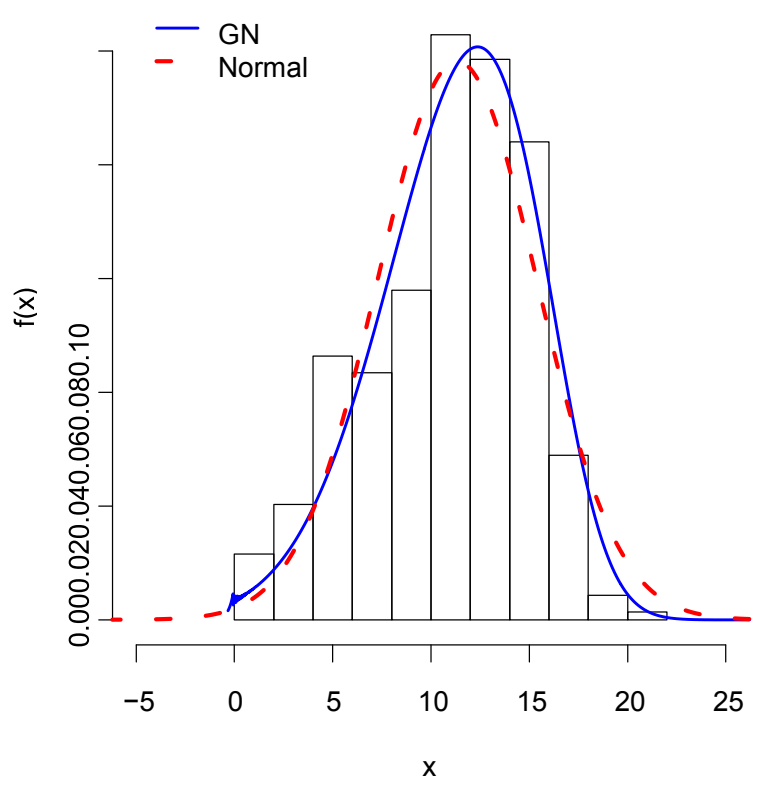

(b)

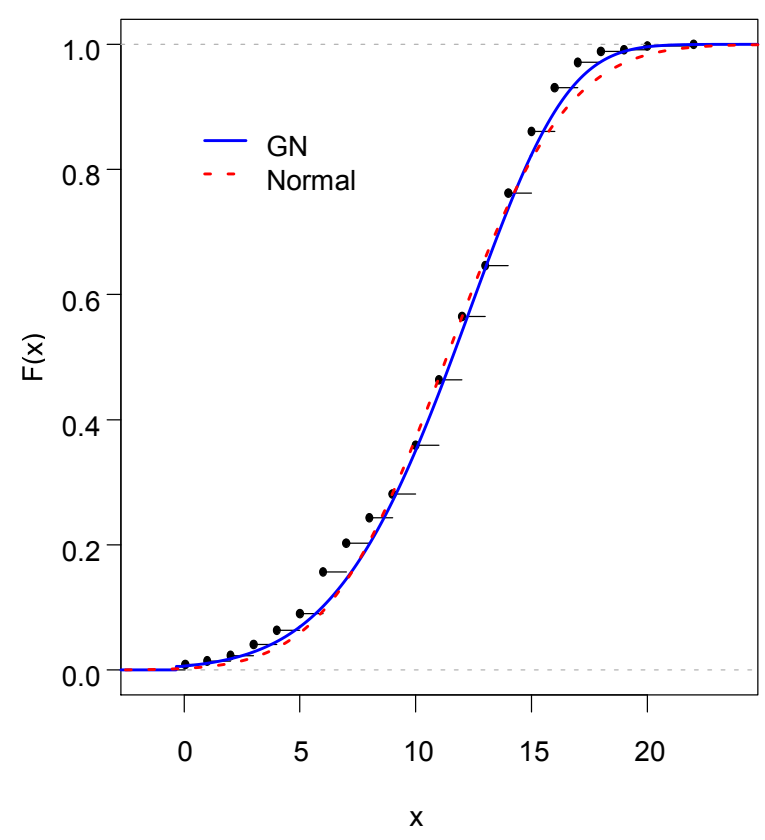

Figure 4: (a) Estimated densities of the GN and normal models for carbon monoxide data. (b) Estimated cumulative functions and the empirical cdf for carbon monoxide data.

\section{Concluding remarks}

In this paper, we propose a new model called the gamma-normal distribution which extends the normal distribution. The proposed distribution is very versatile to fit real data and could be a good alternative to the normal and two recent generalizations of this distribution. We study some of its structural properties. We provide explicit expressions for the ordinary and incomplete moments, quantile and generating functions, mean deviations, Rényi entropy, Shannon entropy, order statistics and their moments. We derive a power series expansion for its quantile function which is useful to obtain alternative formulae for several mathematical measures. The model parameters are estimated by maximum likelihood and the observed information matrix is determined. The potentiality of the new model is illustrated by means of two examples.

\section{Appendix A: Quantile function}

We derive a power series for the QGN(u) in the following way. First, we use a known power series for $\mathrm{Q}-1(\mathrm{a}, 1-\mathrm{u})$. Second, we obtain a power series for the argument $1-\exp [-\mathrm{Q}-1(\mathrm{a}, 1-\mathrm{u})]$. Third, we consider the power series for the normal quantile function given in Steinbrecher (2002) to obtain a power series for QGN(u).

We introduce the following quantities defined by Cordeiro and Lemonte (2011). Let $\mathrm{Q}^{-1}(\mathrm{a}, \mathrm{z})$ be the inverse function of 


$$
Q(a, z)=1-\frac{\gamma(a, z)}{\Gamma(a)}=\frac{\Gamma(a, z)}{\Gamma(a)}=u
$$

The inverse quantile function $Q^{-1}(a, 1-u)$ is determined in the Wolfram website ${ }^{1}$ as

$$
\begin{aligned}
Q^{-1}(a, 1-u) & =w+\frac{w^{2}}{a+1}+\frac{(3 a+5) w^{3}}{2(a+1)^{2}(a+2)}+\frac{[a(8 a+33)+31] w^{4}}{3(a+1)^{3}(a+2)(a+3)} \\
& +\frac{\{a(a[a(125 a+1179)+3971]+5661)+2888\} w^{5}}{24(a+1)^{4}(a+2)^{2}(a+3)(a+4)}+O\left(w^{6}\right),
\end{aligned}
$$

where $w=[u \Gamma(a+1)]^{1 / a}$. We can write the last equation as

$$
z=Q^{-1}(a, 1-u)=\sum_{r=0}^{\infty} \delta_{r} u^{r / a},
$$

where $\delta_{i}^{\prime}$ 's is given by $\delta_{i}=b_{i} \Gamma(a+1)^{i / a}$. Here, $b_{0}=0, b_{1}=1$ and any coefficient $b_{i+1}$ (for $\left.i \geq 1\right)$ can be obtained from the cubic recurrence equation

$$
\bar{b}_{i+1}=\frac{1}{i(a+i)}\left\{\sum_{r=1}^{i} \sum_{s=1}^{i-s+1} \bar{b}_{r} \bar{b}_{s} \bar{b}_{i-r-s+2} s(i-r-s+2) \sum_{r=2}^{i} \bar{b}_{r} \bar{b}_{i-r+2} r[r-a-(1-a)(i+2-r)]\right\} .
$$

The first coefficients are $b 2=1 /(a+1), b 3=(3 a+5) /[2(a+1) 2(a+2)], \ldots$. Now, we present some algebraic details for the GN quantile function, say QGN(u). The cdf of X is given by (6). By inverting $\mathrm{F}(\mathrm{x})=\mathrm{u}$, we obtain (12). The normal quantile function can be expressed as (Steinbrecher, 2002)

$$
Q_{N}(u)=\Phi^{-1}(x)=\sum_{k=0}^{\infty} d_{k}[\sqrt{2 \pi}(u-1 / 2)]^{k},
$$

where the coefficients $d_{k}^{\prime}$ S are defined by $d_{k}=0$ for $k=0,2,4, \ldots$ and $\left.d_{k}=e_{(k}-1\right)_{2}$ for $k=1,3,5, \ldots$ The quantities $e_{k}^{\prime}$ 's are determined recursively from

$$
e_{k+1}=\frac{1}{2(2 k+3)} \sum_{r=0}^{k} \frac{(2 r+1)(2 k-2 r+1) e_{r} e_{k-r}}{(r+1)(2 r+1)} .
$$

Expanding the binomial term in (43), we obtain

$$
Q_{N}(u)=\sum_{k=0}^{\infty}(\sqrt{2 \pi})^{k} d_{k} \sum_{s=0}^{k}(-2)^{s-k}\left(\begin{array}{l}
k \\
s
\end{array}\right) u^{s}=\sum_{k=0}^{\infty} \sum_{s=0}^{k}(\sqrt{2 \pi})^{k}(-2)^{s-k} d_{k}\left(\begin{array}{l}
k \\
s
\end{array}\right) u^{s} .
$$

Changing $\sum_{k=0}^{\infty} \sum_{s=0}^{k}$ by $\sum_{s=0}^{\infty} \sum_{k=s}^{\infty}$, we have

$$
Q_{N}(u)=\sum_{s=0}^{\infty} \sum_{k=s}^{\infty}(\sqrt{2 \pi})^{k}(-2)^{s-k} d_{k}\left(\begin{array}{l}
k \\
s
\end{array}\right) u^{s},
$$

and then $Q_{N}(u)=\sum_{s=0}^{\infty} w_{s} u^{s}$, where $\omega_{s}=\sum_{k=s}^{\infty}(-2)^{s-k}(\sqrt{2 \pi})^{k}\left(\begin{array}{l}k \\ s\end{array}\right) d_{k}$ and the quantity $d_{k}$ was defined above.

\footnotetext{
${ }^{1}$ http://functions.wolfram.com/GammaBetaErf/InverseGammaRegularized/06/01/03/
} 
By replacing (42) in equation (12), we can write

$$
Q_{G N}(u)=\mu+\sigma Q_{N}\left\{1-\exp \left[-\sum_{r=0}^{\infty} \delta_{r} u^{r / a}\right]\right\}
$$

By expanding the exponential function and using (14), we have

$$
\begin{aligned}
1-\exp \left(-\sum_{r=0}^{\infty} \delta_{r} u^{r / a}\right) & =1-\sum_{l=0}^{\infty} \frac{(-1)^{l}\left(\sum_{r=0}^{\infty} \delta_{r} u^{r / a}\right)^{l}}{l !} \\
& =1-\sum_{l=0}^{\infty} \frac{(-1)^{l} \sum_{r=0}^{\infty} f_{l, r} u^{r / a}}{l !}=1-\sum_{r=0}^{\infty} p_{r} u^{r / a},
\end{aligned}
$$

where $_{p_{r}}=\sum_{l=0}^{\infty} \frac{(-1)^{l} f_{l, r}}{l !}, f_{l, r}=\left(r \delta_{0}\right)^{-1} \sum_{q=1}^{r}[q(l+1)-r] \delta_{m} f_{l, r-q}$ for $r \geq 1$ and $f_{l, 0}=\delta_{0}^{l}$. Combining (12) and (44), we obtain

$$
Q_{G N}(u)=\mu+\sigma Q_{N}\left(1-\sum_{r=0}^{\infty} p_{r} u^{r / a}\right) .
$$

Using the know result for $Q_{N}(u)$ in the last equation and expanding the binomial term, we have

$$
Q_{G N}(u)=\mu+\sigma\left\{\sum_{s=0}^{\infty} w_{s}\left(1-\sum_{r=0}^{\infty} p_{r} u^{r / a}\right)^{s}\right\}=\mu+\sigma\left\{\sum_{s=0}^{\infty} w_{s} \sum_{j=0}^{s}(-1)^{j}\left(\begin{array}{l}
s \\
j
\end{array}\right)\left(\sum_{r=0}^{\infty} p_{r} u^{r / a}\right)^{j}\right\}
$$

Now, using (14), we obtain

$$
Q_{G N}(u)=\mu+\sigma\left\{\sum_{s=0}^{\infty} \sum_{j=0}^{s}(-1)^{j}\left(\begin{array}{l}
s \\
j
\end{array}\right) w_{s} \sum_{r=0}^{\infty} h_{j, r} u^{r / a}\right\}=\mu+\sigma\left\{\sum_{s, r=0}^{\infty} \sum_{j=0}^{s}(-1)^{j} w_{s} h_{j, r}\left(\begin{array}{l}
s \\
j
\end{array}\right) u^{r / a}\right\},
$$

Where $h_{j, r}=\left(r p_{0}\right)^{-1} \sum_{m=0}^{r}[m(j+1)-r] p_{m} h_{j, r-m}$. Finally,

$$
Q_{G N}(u)=\mu+\sigma \sum_{r=0}^{\infty} \bar{p}_{j} h_{j, r} u^{r / a}
$$

Where $\overline{\mathrm{p}_{J}}=\sum_{s=0}^{\infty} \sum_{j=0}^{S}(-1)^{j} \omega_{s}\left(\begin{array}{l}S \\ j\end{array}\right)$

\section{Appendix B: Moments}

Here, we use equation (14) and the power series $\Phi(x)=\sum_{j=0}^{\infty} a_{j} x^{j}$ given in Section 5. We have

$$
T_{n}(y)=\int_{0}^{y} x^{n} f(x) d x .
$$

Inserting (11) (with $\mu=0$ and $\sigma=1$ ) in the last equation gives

$$
T_{n}(y)=\sum_{r=0}^{\infty} d_{r} \int_{0}^{y} x^{n} \phi(x) \Phi(x)^{r} d x .
$$

From the power series for $\Phi(x)$ and equation (14), we have

$$
T_{n}(y)=\sum_{j, r=0}^{\infty} d_{r} c_{r, j} \int_{0}^{y} x^{n+j} \phi(x) d x=\frac{1}{\sqrt{2 \pi}} \sum_{j, r=0}^{\infty} d_{r} c_{r, j} \int_{0}^{y} x^{n+j} \mathrm{e}^{-\mathrm{x}^{2} / 2} \mathrm{dx},
$$


where dr is defined in Section 3 and the quantities cr,j are obtained from (15) using the ai's of the power series for $\Phi(\mathrm{x})$. Setting $\mathrm{z}=\mathrm{x} 2 / 2$, we obtain

$$
T_{n}(y)=\frac{1}{\sqrt{2 \pi}} \sum_{j, r=0}^{\infty} d_{r} c_{r, j} \int_{0}^{y^{2} / 2}(2 z)^{\frac{n+j-1}{2}} \mathrm{e}^{-\mathrm{z}} \mathrm{dz}=\frac{1}{\sqrt{2 \pi}} \sum_{\mathrm{j}, \mathrm{r}=0}^{\infty} 2^{\mathrm{n}+\mathrm{j}-1} \mathrm{~d}_{\mathrm{r}} \mathrm{c}_{\mathrm{r}, \mathrm{j}} \gamma\left(\frac{\mathrm{n}+\mathrm{j}+1}{2}, \frac{\mathrm{y}^{2}}{2}\right),
$$

where $\gamma(\cdot, \cdot)$ is the gamma incomplete function.

The second representation for $\operatorname{Tn}(\mathrm{y})$ is based on the integral $A(j, q)=\int_{-\infty}^{q} x^{j} \mathrm{e}^{-\mathrm{x}^{2} / 2} \mathrm{dx}$, which is determined for $\mathrm{q}>0$ and $\mathrm{q}<0$. We define

$$
G(j)=\int_{0}^{\infty} x^{j} \mathrm{e}^{-\mathrm{x}^{2} / 2} \mathrm{dx}=2^{(\mathrm{j}-1) / 2} \Gamma\left(\frac{\mathrm{j}+1}{2}\right) .
$$

For $\mathrm{q}<0$ and $\mathrm{q}>0$, we have

$$
A(j, q)=(-1) j G(j)+(-1) j+1 H(j, q) \quad \text { and } A(j, q)=(-1) j G(j)+H(j, q) \text {, }
$$

respectively, where the integral $H(j, q)=\int_{0}^{j} x^{j} \mathrm{e}^{-\mathrm{x}^{2} / 2} \mathrm{dx}$ can be easily determined as (Whittaker and Watson, 1990)

$$
\begin{aligned}
H(j, q) & =\frac{2^{j / 4+1 / 4} q^{j / 2+1 / 2} e^{-q^{2} / 4}}{(j / 2+1 / 2)(j+3)} N_{j / 4+1 / 4, j / 4+3 / 4}\left(q^{2} / 2\right) \\
& +\frac{2^{j / 4+1 / 4} q^{j / 2-3 / 2} e^{-q^{2} / 4}}{j / 2+1 / 2} N_{j / 4+5 / 4, j / 4+3 / 4}\left(q^{2} / 2\right),
\end{aligned}
$$

where $N_{k, m}(x)$ is the Whittaker function (Abramowitz and Stegun, 1972, p. 505; Whittaker and Watson 1990, pp. 339-351) given, in terms of the confluent hypergeometric function $\mathrm{F}_{1}(a ; b ; z)=\sum_{k=0}^{\infty} \frac{(a) k}{(b) k} \frac{z^{k}}{k !}$, or in terms of the Kummer's function $U(a, b ; z)=z^{-a}{ }_{2} F_{0}\left(a, 1+a-b ;-z^{-1}\right)$, where $(a)_{k}$ was defined inSection 5 . We have

$$
N_{k, m}=\frac{x^{m+1 / 2}}{\mathrm{e}^{-\mathrm{x} / 2}}{ }_{1} F_{1}\left(\frac{1}{2}+m-k, 1+2 m ; x\right) \quad \text { and } \quad \mathrm{N}_{\mathrm{k}, \mathrm{m}}=\frac{\mathrm{x}^{\mathrm{m}+1 / 2}}{\mathrm{e}^{-\mathrm{x} / 2}} \mathrm{U}\left(\frac{1}{2}+\mathrm{m}-\mathrm{k}, 1+2 \mathrm{~m} ; \mathrm{x}\right) .
$$

Combining (11) and (21), we can write

$$
\begin{gathered}
T_{n}(y)=\frac{1}{\sqrt{2 \pi}} \sum_{k, r=0}^{\infty} b_{k} s_{r+1}(a+k) \int_{0}^{y} x^{n} \mathrm{e}^{-\mathrm{x}^{2} / 2} \sum_{\mathrm{j}=0}^{\infty} \mathrm{cr}, \mathrm{xj} \mathrm{dx} \\
=\frac{1}{\sqrt{2 \pi}} \sum_{j, k, r=0}^{\infty} b_{k} s_{r+1}(a+k) c_{r, j} \int_{-\infty}^{y} x^{j+n} \mathrm{e}^{-\mathrm{x}^{2} / 2} \mathrm{dx},
\end{gathered}
$$

where $_{c_{r, j}}=\left(j a_{0}\right)^{-1} \sum_{m=1}^{j}[m(r+1)-j] a_{m} c_{r, j-m}$, for $j \geq 1, c_{r, 0}=a_{0}^{r}$ and $c_{0,0}=1$ and the quantities $a_{i}^{\prime}$ 's are defined in Section 5.

Computing the last integral, we can write

$$
T_{n}(y)=\frac{1}{\sqrt{2} \pi} \sum_{k, r, j=0}^{\infty} b_{k} c_{r, j} s_{r+1}(a+k) A(j+n, y)
$$

where $A(\cdot, \cdot)$ is determined as before and $s_{r}(a)$ is given by $(10)$.

The third representation for $T_{n}(y)$ is based on the normal quantile function. We have

$$
T_{n}(y)=\sum_{r=0}^{\infty} d_{r} \int_{-\infty}^{y} x^{n} \phi(x) \Phi(x)^{r} d x
$$


The last integral can be rewritten according to the normal quantile function QN(u) given in Section4.Thus, using equations (14) and (13), we have

$$
T_{n}(y)=\sum_{r=0}^{\infty} d_{r} \int_{0}^{\Phi(y)}\left(\sum_{s=0}^{\infty} w_{s} u^{s}\right)^{n} u^{r} d u=\sum_{r=0}^{\infty} d_{r} \int_{0}^{\Phi(y)} \sum_{s=0}^{\infty} e_{n, s} u^{r+s} d u
$$

where $_{e_{n, s}}=\left(s w_{0}\right)^{-1} \sum_{m=1}^{s}[m(n+1)-s] w_{m} e_{n, s-m}($ for $s \geq 1), e_{n, 0}=w_{0}^{n}$ and the quantities $w_{m}$ 's are given in Section 4. Finally, we obtain

\section{Appendix C: Generating function}

$$
T_{n}(y)=\sum_{r, s=0}^{\infty} d_{r} e_{n, s} \frac{\Phi(y)^{r+s+1}}{(r+s+1)}
$$

Here, we present the algebraic details of the second representation for $\mathrm{M}(\mathrm{t})$ based on the quantile power series of X. Using (16) with $\mu=0$ and $\sigma=1$, we obtain

$$
M(t)=\int_{0}^{1} \exp \left[t Q_{G N}(u)\right] d u=\int_{0}^{1} \exp \left[t\left(\sum_{r=0}^{\infty} \bar{p}_{j} h_{j, r} u^{r / a}\right)\right] d u,
$$

Where $\overline{\mathrm{p}_{j}}=\sum_{s=0}^{\infty} \sum_{j=0}^{s}(-1)^{j}\left(\begin{array}{l}S \\ j\end{array}\right) \omega_{s}, \omega_{s}=\sum_{k=s}^{\infty}(\sqrt{2 \pi})^{k}(-2)^{s-k} d_{k}\left(\begin{array}{l}k \\ S\end{array}\right)$ and $h_{j, i}=\left(i \mathrm{p}_{0}\right)^{-1} \sum_{m=0}^{i}[m(j+1)-i] p_{m}$ $h_{j, i}-m$. Other quantities are well-defined in Section 4.

Expanding the exponential function, we have

$$
M(t)=\int_{0}^{1} \sum_{k=0}^{\infty} \frac{t^{k}\left(\sum_{r=0}^{\infty} \bar{p}_{j} h_{j, r} u^{r / a}\right)^{k}}{k !} d u=\sum_{k, r=0}^{\infty} \frac{d_{k, r}}{\left(\frac{r}{a}+1\right)} \frac{t^{k}}{k !}
$$

where $d_{k, r}=\left(r g_{0}\right)^{-1} \sum_{m=1}^{r}[m(k+1)-r] g_{m} d_{k, r-m}$ (for $\left.r \geq 1\right), d_{k, 0}=g_{0}^{r}, d_{0,0}=1$, the quantities $g_{j}$ 's are given by $g_{j}=\overline{p_{j}} h_{j, r}$ and the other quantities $p_{j}$ and $h_{j, r}$ are defined before.

\section{Appendix D: Rényi entropy}

The Rényi entropy of a random variable with pdf $\mathrm{f}(\mathrm{x})$ is defined as

$$
I_{R}(\gamma)=\frac{1}{1-\gamma} \log \int_{-\infty}^{+\infty} f^{\gamma}(x) d x
$$

for $\gamma>0$ and $\gamma \neq 1$. We provide details about the Rényi entropy for $\gamma$ positive integer first and then for positive real.

First, assuming $\gamma=\mathrm{n}=2,3, \ldots, \mu=0$ and $\sigma=1$, we can write from (11) and (21) 


$$
\begin{aligned}
I_{R}(n) & =\frac{1}{1 \mid-n} \log \int_{-\infty}^{+\infty}\left(\frac{1}{\sqrt{2 \pi}} \mathrm{e}^{-\mathrm{x}^{2} / 2} \sum_{\mathrm{r}=0}^{\infty} \mathrm{d}_{\mathrm{r}} \Phi(\mathrm{x})^{\mathrm{r}}\right)^{n} d x \\
& =\frac{1}{1-n} \log \int_{-\infty}^{+\infty}\left\{\left(\frac{1}{\sqrt{2 \pi}}\right)^{n} \mathrm{e}^{-\mathrm{nx} \mathrm{x}^{2} / 2}\left(\sum_{\mathrm{r}=0}^{\infty} \mathrm{d}_{\mathrm{r}} \sum_{\mathrm{j}=0}^{\infty} \mathrm{c}_{\mathrm{r}, \mathrm{j}} \mathrm{x}^{\mathrm{j}}\right)^{\mathrm{n}} \mathrm{dx}\right\} \\
& =\frac{1}{1-n} \log \int_{-\infty}^{+\infty}\left\{\left(\frac{1}{\sqrt{2 \pi}}\right)^{n} \mathrm{e}^{-\mathrm{nx} \mathrm{x}^{2} / 2}\left(\sum_{\mathrm{j}=0}^{\infty} \overline{\mathrm{e}}_{\mathrm{j}} \mathrm{x}^{\mathrm{j}}\right)^{\mathrm{n}} \mathrm{dx}\right\} \\
& =\frac{1}{1-n} \log \left\{\left(\frac{1}{\sqrt{2 \pi}}\right)^{n} \sum_{j=0}^{n} \wp_{n, j} \int_{-\infty}^{+\infty} x^{j} \mathrm{e}^{-\frac{\mathrm{nx}}{2}} \mathrm{dx}\right\}
\end{aligned}
$$

Letting $\mathrm{y}=\sqrt{ } \mathrm{nx}$ and using equation (29), we have

$$
I_{R}(n)=\frac{1}{n-1}\left\{\frac{(n-1)}{2} \log (2 \pi)+\frac{(j+1)}{2} \log (n)-\log \left[\sum_{j=0}^{n} \wp_{n, j} m_{j}^{\prime}\right]\right\},
$$

where $\wp_{n, j}=\left(j \bar{e}_{0}\right)^{-1} \sum_{m=1}^{j}[m(n+1)-j] e_{m} \wp_{n, j-m}$ (for $\left.j \geq 1\right), \wp_{n, 0}=e_{0}^{n}, e_{j}=\sum_{j=0}^{\infty} d_{r} c_{r, j}$ and $\mathrm{m}^{\prime} \mathrm{j}$ is the jth moment of the normal distribution. The quantities dr's are defined in Section 3 and the av's and cr,j's are given in Section 5.

We can write $\operatorname{IR}(\gamma)=(1-\gamma)-1 \mathrm{E}\{\mathrm{f}(\mathrm{Z}) \gamma-1\}$. Let $\delta=\mathrm{E}(\mathrm{Z})$. For $\gamma$ real positive, we can write

$$
\mathrm{E}\{\mathrm{f}(\mathrm{Z}) \gamma-1\}=\delta \gamma-1 \mathrm{E}\{1+\theta[\mathrm{f}(\mathrm{Z})-\delta]\} \gamma-1,
$$

where $\theta=\delta-1$. From the generalized binomial expansion, we obtain

$$
\{1+\theta[f(Z)-\delta]\}^{\gamma-1}=1+\sum_{n=1}^{\infty} \frac{\theta^{n} \Im_{n}}{n !}[f(Z)-\delta]^{n},
$$

where $^{\Im_{n}}=\prod_{j=0}^{n-1}(\gamma-1-j)$. Further, we have

$$
E\left\{f(Z)^{\gamma-1}\right\}=\delta^{\gamma-1}\left(1+\sum_{n=2}^{\infty} \frac{\theta^{n} \Im_{n}}{n !} E\left\{[f(Z)-\delta]^{n}\right\}\right) .
$$

We now calculate $\mathrm{E}\{[\mathrm{f}(\mathrm{Z})] \mathrm{n}\}$ for $\mathrm{n} \geq 2$. From equation (11) and using the binomial expansion, we can write

$$
\rho_{n}=E\left\{[f(Z)]^{n}\right\}=\sum_{j=0}^{\infty} \wp_{n, j} \psi_{n, j}
$$

where $\psi n, j=E\{Z j \phi(Z) n\}$. Then,

$$
\psi_{n, j}=\int_{-\infty}^{\infty} x^{j} \phi(x)^{n+1} \mathrm{dx}
$$

Setting $\sqrt{(\mathrm{n}+1) \mathrm{x}}=\mathrm{y}$, we can easily determine the last integral and write $\rho n$ as

$$
\rho_{n}=\left(\frac{1}{\sqrt{2 \pi}}\right)^{n} \sum_{j=0}^{\infty} \wp_{n, j}\left(\frac{1}{\sqrt{n+1}}\right)^{j+1} m_{j}^{\prime} .
$$


By expanding the binomial term in (31), we can obtain an explicit expression for $\operatorname{IR}(\gamma)$, which holds for any $\gamma$ real positive and $\gamma=1$, given by

$$
I_{R}(\gamma)=(1-\gamma)^{-1} \delta^{\gamma-1}\left[1+\sum_{n=2}^{\infty} \frac{\theta^{n} \Im_{n}}{n !} \sum_{k=0}^{n}(-\delta)^{n-k}\left(\begin{array}{l}
n \\
k
\end{array}\right) \rho_{k}\right],
$$

where $\rho \mathrm{j}$ is determined from (32).

\section{Appendix E: The observed information matrix}

The elements of the observed information matrix $J(\theta)$ for the three parameters $(a, \mu, \sigma)$ are given by:

$$
\begin{gathered}
J_{a a}=-n \psi^{\prime}(a), \quad J_{a \mu}=\frac{1}{\sigma} \sum_{i=1}^{n} \frac{\phi\left(z_{i}\right)}{\left[1-\Phi\left(z_{i}\right)\right] \log \left[1-\Phi\left(z_{i}\right)\right]}, \quad J_{a \mu}=\frac{1}{\sigma} \sum_{i=1}^{n} \frac{z_{i} \phi\left(z_{i}\right)}{\left[1-\Phi\left(z_{i}\right)\right] \log \left[1-\Phi\left(z_{i}\right)\right]}, \\
J_{\mu \mu}=-\frac{n}{\sigma^{2}}+\frac{(a-1)}{\sigma^{2}} \sum_{i=1}^{n} \frac{z_{i} \phi\left(z_{i}\right)}{\left[1-\Phi\left(z_{i}\right)\right] \log \left[1-\Phi\left(z_{i}\right)\right]}-\frac{(a-1)}{\sigma} \sum_{i=1}^{n} \frac{\phi^{2}\left(z_{i}\right)\left\{1+\log \left[1-\Phi\left(z_{i}\right)\right]\right\}}{\left[1-\Phi\left(z_{i}\right)\right]^{2}\left\{\log \left[1-\Phi\left(z_{i}\right)\right]\right\}^{2}}, \\
J_{\mu \sigma}=-\frac{2}{\sigma^{2}} \sum_{i=1}^{n} z_{i}+\frac{(a-1)}{\sigma^{2}} \sum_{i=1}^{n} \frac{\left(z_{i}^{2}-1\right) \phi\left(z_{i}\right)}{\left[1-\Phi\left(z_{i}\right)\right] \log \left[1-\Phi\left(z_{i}\right)\right]}-\frac{(a-1)}{\sigma^{2}} \sum_{i=1}^{n} \frac{z_{i} \phi^{2}\left(z_{i}\right)\left\{1+\log \left[1-\Phi\left(z_{i}\right)\right]\right\}}{\left[1-\Phi\left(z_{i}\right)\right]^{2}\left\{\log \left[1-\Phi\left(z_{i}\right)\right]\right\}^{2}}, \\
J_{\sigma \sigma}=\frac{n}{\sigma^{2}}-\frac{3}{\sigma^{2}} \sum_{i=1}^{n} z_{i}^{2}+\frac{2(a-1)}{\sigma^{2}} \sum_{i=1}^{n} \frac{z_{i} \phi\left(z_{i}\right)}{\left[1-\Phi\left(z_{i}\right)\right] \log \left[1-\Phi\left(z_{i}\right)\right]}-\frac{(a-1)}{\sigma^{2}} \sum_{i=1}^{n} \frac{z_{i}^{2} \phi^{2}\left(z_{i}\right)\left\{1+\log \left[1-\Phi\left(z_{i}\right)\right]\right\},}{\left[1-\Phi\left(z_{i}\right)\right]^{2}\left\{\log \left[1-\Phi\left(z_{i}\right)\right]\right\}^{2}}
\end{gathered}
$$

where $z_{i}=\left({ }^{x i} \sigma^{-\mu}\right)$ and $\psi^{\prime}(\cdot)$ is the trigamma function.

\section{References}

[1] Alexander, A., Cordeiro, G.M., Ortega, E.M.M. and Sarabia, J.M. (2012). Generalized betagenerated distributions. Computational Statistics and Data Analysis , 56, 1880-1897.

[2] Azzalini, A. (1985). A class of distributions which includes the normal ones. Scandinavian Journal of Statistics, 12, 171-178.

[3] Abramowitz, M. and Stegun, I. A. (1972). "Confluent Hypergeometric Functions". Ch. 13 in Handbook of Mathematical Functions with Formulas, Graphs, and Mathematical Tables. Dover, New York. 503-515.

[4] Barakat, H.M. and Abdelkader, Y.H. (2004). Computing the moments of order statistics from nonidentical random variables. Statistical Methods and Application, 13, 15-26.

[5] Cordeiro, G.M., Ortega, E.M.M. and Silva, G.O. (2011). The exponentiated generalized gamma distribution with application to lifetime data. Journal of Statistical Computation and Simulation, 81, 827-842.

[6] Cordeiro, G.M., Cintra, R.J., Rêgo, L.C and Ortega, E.M.M. (2012). The McDonald normal distribution. Pakistan Journal of Statistics and Operation Research, 8, 301-329.

[7] Cordeiro, G.M. and Lemonte, A.J. (2011). The $\beta$-Birnbaum-Saunders distribution: An improved distribution for fatigue life modeling.Computational Statistics and Data Analysis, 55, 1445-1461.

[8] Doornik, J.A. (2006). An Object-Oriented Matrix Language - Ox 4, 5th ed. Timberlake Consultants Press, London. 
[9] Eugene, N., Lee, C. and Famoye, F. (2002). Beta-normal distribution and its applications. Communications in Statistics: Theory and Methods, 31, 497-512.

[10] Exton, H. (1978). Handbook of Hypergeometric Integrals: Theory, Applications, Tables, Computer Programs. Halsted Press, New York.

[11] Gradshteyn, I.S. and Ryzhik, I.M. (2007). Table of integrals, series, and products. Academic Press, New York.

[12] Gupta, R.D. and Kundu, D. (2001). Exponentiated Exponential Family: An Alternative to Gamma and Weibull Distributions. Biometrical Journal, 43, 117-130.

[13] Gupta, R.C., Gupta, P.L. and Gupta, R.D. (1998). Modeling failure time data by Lehman alternatives. Communications Statistics - Theory and Methods, 27, 887-904.

[14] Matsuo, T. 0. (1986). Uso da regressão de cumeeira em esperimentos agronômicos. Master Thesis, Escola Superior de Agricultura “Luiz de Queiroz”, University of São Paulo, Brasil (in Portuguese).

[15] Mudholkar, G.S. and Srivastava, D.K. (1993). Exponentiated Weibull family for analyzing bathtub failure-real data. IEEE Transaction on Reliability, 42, 299-302.

[16] Mudholkar, G.S., Srivastava, D.K. and Friemer, M. (1995). The exponential Weibull family: A reanalysis of the bus-motor failure data. Technometrics, 37, 436-445.

[17] Nadarajah, S. (2008). Explicit expressions for moments of order statistics. Statistics and Probability Letters, 78, 196-205.

[18] Nadarajah, S., Cordeiro, G.M. and Ortega, E.M.M. (2013). The gamma-G family of distributions. Mathematical properties and applications. To appear in Communication in Statistics. Theory Methods.

[19] Nadarajah, S. and Gupta, A.K. (2007). A generalized gamma distribution with application to drought data. Mathematics and Computer in Simulation, 74, 1-7.

[20] Prudnikov, A.P., Brychkov, Y.A. and Marichev, O.I. (1986). Integrals and series: Elementary functions. Gordon \& Breach Science Publishers, New York.

[21] Rényi, A. (1961). On measures of information and entropy. Proceedings of the 4th Berkeley Symposium on Mathematics, Statistics and Probability 1960. 547-561.

[22] Ristic, M.M. and Balakrishnan, N. (2011). The gamma exponentiated exponential distribution. Journal of Statistical Computation and Simulation, doi: 10.1080/00949655.2011.574633.

[23] Shanon, C.E. (1951). Prediction and Entropy of Printed English. Bell System Technical Journal, 30, 50-64.

[24] Steinbrecher, G. (2002). Taylor expansion for inverse error function around origin. (Working paper at University of Craiova).

[25] Weisberg, S. (2005). Applied linear regression (3rd ed.). New York: Wiley.

[26] Whittaker, E.T. and Watson, G.N. (1990). A Course in Modern Analysis. Cambridge University Press, Cambridge.

[27] Zografos, K. and Balakrishnan, N. (2009). On families of beta- and generalized gamma-generated distributions and associated inference. Statistical Methodology, 6, 344-362. 